\title{
String Field Theory Around the Tachyon Vacuum
}

\author{
Leonardo Rastelli ${ }^{a},{\text { Ashoke } \text { Sen }^{b} \text { and Barton Zwiebach }}^{c}$
}

\author{
${ }^{a}$ Department of Physics \\ Princeton University, Princeton, NJ 08540 \\ rastelli@feynman.princeton.edu
}

${ }^{b}$ Harish-Chandra Research Institute ${ }^{1}$

Chhatnag Road, Jhusi, Allahabad 211019, INDIA

asen@thwgs.cern.ch, sen@mri.ernet.in

${ }^{c}$ Center for Theoretical Physics

Massachussetts Institute of Technology,

Cambridge, MA 02139, USA

zwiebach@mitlns.mit.edu

\begin{abstract}
Assuming that around the tachyon vacuum the kinetic term of cubic open string field theory is made purely of ghost operators we are

e-print archive: http://xxx.lanl.gov/hep-th/001225

${ }^{1}$ Formerly Mehta Research Institute of Mathematics and Mathematical Physics
\end{abstract}


led to gauge invariant actions which manifestly implement the absence of open string dynamics around this vacuum. We test this proposal by showing the existence of lump solutions of arbitrary codimension in this string field theory. The key ingredients in this analysis are certain assumptions about the analyticity properties of tachyon Green's functions. With the help of some further assumptions about the properties of these Green's functions, we also calculate the ratios of tensions of lump solutions of different dimensions. The result is in perfect agreement with the known answers for the ratios of tensions of D-branes of different dimensions.

\section{Contents}

1 Introduction and Summary

2 String Field Theory Around the Tachyon Vacuum

2.1 Algebraic structure before and after condensation . . . . . . . 360

2.2 An ansatz for the SFT after condensation . . . . . . . . . 361

3 The Lump Solutions and Their Tensions 365

3.1 Analysis of the tachyon effective action . . . . . . . . 365

3.2 Setup for lump solutions . . . . . . . . . . . . . 368

3.3 Equations for lump solutions . . . . . . . . . . . . 370

3.4 Solving the lump equations ... . . . . . . . . . 373

3.5 Computation of ratios of lump tensions . . . . . . . . 376

4 Further studies and open questions $\quad 379$

4.1 On the uniqueness of $\mathcal{Q} \ldots \ldots 379$

4.2 On the use of level truncation . . . . . . . . . . . 381

4.3 On gauge fixing . . . . . . . . . . . . . . . . 382

4.4 On superstring field theory and closed strings . . . . . . . . . 384

4.5 Concluding remarks . . . . . . . . . . . . . 385 


\section{Introduction and Summary}

The 26-dimensional bosonic string theory contains D-p-branes for all integer $p \leq 26$. Each of these D-p-branes has a tachyonic mode. It has been conjectured $[1,2]$ that there is a local minimum of the tachyon potential which describes the closed string vacuum without any D-brane. At this minimum the negative contribution from the tachyon potential exactly cancels the tension of the D-brane. Furthermore, since the brane disappears, this vacuum cannot support conventional open string excitations. It has also been conjectured that a codimension $q$ lump solution on the $\mathrm{D}$ - $p$-brane represents a $\mathrm{D}-(p-q)$-brane in the same theory. Support for these conjectures comes from the analysis of the world-sheet theory $[1,3,4,5,6,7,8]$, cubic open string field theory $[9,10,11,12,13,14,15,16,17,19,20,21,22,23,24]$, noncommutative limit of the effective field theory of the tachyon [25, 26, 27, 28, 29, 30], background independent open string field theory [31,32, 33, 34], as well as various simple models of tachyon condensation [35, 36, 37, 38, 39, 40].

One of the promising approaches to the study of this subject matter involves the use of cubic open string field theory (SFT) [41]. Indeed, much work has already been done with it using the level truncation scheme $[9,10,11]$. While complete analytic solutions of the equations of motion of this string field theory are still missing, the solutions found by combination of analytic and numerical work gave some of the strongest evidence for the conjectures, as well as demonstrated that string field theory contains nonperturbative physics. More recently, work in boundary string field theory (B-SFT) [42, 43, 44, 45, 46, 47], - a version of string field theory incorporating a certain degree of background independence, - confirmed the form of the tachyon potentials proposed in $[37,38]$ and established conclusively the energetics aspect of the tachyon conjectures [31, 32,33]. Whereas in conventional SFT the tachyon string field condenses, namely an infinite number of scalars acquire expectation values, in B-SFT only the tachyon field acquires a vacuum expectation value. This is quite fortunate, as it appears to be very difficult to formulate B-SFT in generality. Due to this problem in B-SFT, at present cubic string field theory seems to be the only complete framework where we can analyze concretely the fate of the open string field excitations around the vacuum where the D-brane has disappeared. Indeed, it seems to provide a completely non-singular framework to study the tachyon vacuum. Starting from a D-brane background one appears to reach the tachyon vacuum with room to spare and without any indications of singularities in field variables.

In principle the analysis of the tachyon vacuum is straightforward. One 
must: (i) find the classical solution $\Phi_{0}$ representing the vacuum with no D-brane, (ii) expand the string field action setting $\Phi=\Phi_{0}+\Psi$, where $\Psi$ is the fluctuation field, and (iii) analyze the spectrum of $\Psi$ using the resulting kinetic term. Nevertheless, in practice this has not been simple to carry out, first and foremost because there is no known closed form expression for $\Phi_{0}$ yet. The accurate numerical approximations to $\Phi_{0}$ may allow, however, an analysis of the spectrum around the vacuum $[17,18]$.

In this paper we shall analyze the problem from a different angle. Instead of trying to construct the classical solution $\Phi_{0}$, expand the SFT action around $\Phi_{0}$, and attempt field redefinitions to bring the kinetic term to a simple form, we shall make an inspired guess about the form of the SFT action expanded around the tachyon vacuum. Then we will try to check that this action satisfies the various consistency requirements. Since the string field theory action is cubic in the string field, shifting the string field by a classical solution does not change the cubic interaction term. Thus we only need to guess the quadratic term of the shifted action. The requirement that the new action is obtained from the original one by a shift of the string field by a classical solution of the equations of motion puts constraints on the possible choices of the quadratic term, - these basically correspond to the requirement that the shifted action also has an infinite parameter gauge invariance like the original SFT action. Besides these constraints the action must satisfy the following additional requirements:

1. The kinetic operator must have vanishing cohomology. This would imply absence of physical open string states around the tachyon vacuum.

2. The action must have classical solutions representing the original Dbrane configuration, as well as lump solutions representing D-branes of all lower dimensions.

Indeed, our work began with the simple realization that the reparametrization ghost zero mode $c_{0}$ was an obvious replacement for the BRST operator that would lead to vanishing cohomology, and thus no physical states. ${ }^{2}$ More striking, however, is the fact that the Riemann surface description of the star product makes it evident that $c_{0}$ is a derivation. Thus, we noted that replacing the BRST operator $Q_{B}$ by $\mathcal{Q}=c_{0}$ would lead to a string field action

$$
\mathcal{S}(\Psi) \equiv-\frac{1}{g_{0}^{2}}\left[\frac{1}{2}\langle\Psi, \mathcal{Q} \Psi\rangle+\frac{1}{3}\langle\Psi, \Psi * \Psi\rangle\right], \quad \mathcal{Q}=c_{0},
$$

\footnotetext{
${ }^{2}$ This possibility may have occurred to many physicists, but we heard it first from E. Witten [48].
} 
that would have the expected (vanishing) spectrum and would be gauge invariant. It is straightforward to see that more general operators lead to the same conclusions. In fact

$$
\mathcal{Q}=\sum_{n=0}^{\infty} a_{n} \mathcal{C}_{n}, \quad \mathcal{C}_{n} \equiv c_{n}+(-1)^{n} c_{-n},
$$

with arbitrary constants $a_{n}$ 's would also lead to gauge invariant actions with no physical states. As we will show, many of these actions are related by field redefinitions.

In fact, long time ago, Horowitz et.al. [49] constructed a class of formal solutions of the purely cubic string field theory action [50,51] which lead to actions of the above form, with an extra regularity condition $\mathcal{Q}|\mathcal{I}\rangle=0$, where $|\mathcal{I}\rangle$ is the (formal) identity of the star algebra. In particular, these authors noticed that such string field theory would describe no physical excitations around the vacuum in question. Furthermore, since a subclass of the above actions is obtained by shifting the string field in the purely cubic action, which in turn is obtained by shifting the string field in the original SFT action, we can, at least formally, regard the above actions as the result of shifting the string field in the original SFT action. ${ }^{3}$

We will not be able to determine here the precise form and normalization of $\mathcal{Q}$ and we take the above class of actions as confimation that the conditions of zero cohomology, gauge invariance and universality of the kinetic operator can be satisfied. There may even be more general forms of kinetic operators built purely from the ghost sector and satisfying these conditions. Such kinetic terms make the universality of the action expanded around the tachyon vacuum manifest. Indeed, since the tachyon vacuum, representing the closed string vacuum without any D-brane, should not depend on which D-brane configuration we start from, we should expect that the string field theory action expanded around this vacuum should lose all knowledge about the matter conformal field theory (CFT) describing the original D-brane configuration. ${ }^{4}$

Given the class of actions exemplified by eqns. (1.1) and (1.2) the non-

\footnotetext{
${ }^{3}$ These solutions, however, have the unwanted feature that at least formally they have the same energy density as the original D-brane configuration. This is not what is expected of the solution describing the tachyon vacuum. This would suggest that we only consider those $\mathcal{Q}$ 's which $d o$ not satisfy the regularity condition $\mathcal{Q}|\mathcal{I}\rangle=0$. In fact, $c_{0}|\mathcal{I}\rangle \neq 0$.

${ }^{4}$ Since the interaction term involves correlation function in the combined matter-ghost CFT, it does depend on the matter CFT. For this CFT we use the canonical choice corresponding to the maximal dimension space-filling D-brane. However, since the three string vertex involves complete overlap of the three strings the interaction can be made formally background independent by a rescaling of the string fields.
} 
trivial part of the problem is to construct the classical solutions in this string field theory representing the original D-brane as well as various lump solutions representing the lower dimensional D-branes, and compare their tensions with the known tensions of the D-branes. At present we have only been able to partially determine the space-time dependence of the solutions and to calculate the ratio of the tension of a $p$-brane lump to that of a $(p+1)$ brane lump for arbitrary integer $p$. Under certain assumptions which will be explained in section 3 , this computation can be done without a detailed knowledge of the quadratic term in the action as long as it does not involve any matter sector operator. The final result for the ratio of tensions involve certain function $B(\alpha)$ (defined in eqs.(3.34), (3.37), (3.40)) which, if zero, will produce the desired value. Although we cannot at present give an analytic proof of the vanishing of this function, we have checked numerically that this function indeed vanishes to very high degree of accuracy. This in turn is evidence that our guess about the SFT action expanded around the tachyon vacuum is the correct one.

The strategy that we follow for this computation is as follows. Since the kinetic term and hence the propagator does not involve any matter operator we are able to find the exact momentum dependence of an arbitrary $n$-tachyon Green's function. From the generating functional $W[J]$ of the tachyon Green's functions we can find the tachyon effective action by Legendre transformation. Missing the overall ( $n$-dependent) normalization of the $n$-tachyon Green's function, we only have a partial knowledge of the effective action. We now assume that the tachyon equations of motion derived from this effective action have a space-time independent classical solution which can be identified with the original D-brane. Using this assumption, together with certain other assumptions about the analyticity of the generating functional of the tachyon Green's function stated in section 3, we show that the tachyon equation of motion also has lump solution of every codimension. Some further assumptions allow us to calculate the ratios of tensions of these lumps without knowing the detailed form of the effective potential. As already stated, the result is in perfect agreement with the known answers for the ratios of tensions of D-branes.

Clearly many things remain to be done. First and foremost is to reproduce correctly the overall normalization of the D-brane tension. For this we need to fix unambiguously the form of the kinetic term $\mathcal{Q}$ in the shifted vacuum. ${ }^{5}$ Some aspects of our analyticity assumptions may require more information about $\mathcal{Q}$. The action around the tachyon vacuum is expected to

\footnotetext{
${ }^{5}$ While $\mathcal{Q}=0$, leading to the purely cubic action [50], is universal, the resulting background is not suitable for the present analysis since with vanishing kinetic term the Feynman rules used for computing the tachyon effective action are not well defined. In addition,
} 
possess many novel properties. In particular many of the symmetries which are spontaneously broken in the background of a D-brane are expected to be restored in this vacuum $[52,53,54]$. It will be interesting to analyse our proposal to check these properties. Another important problem is to generalize this analysis to the case of superstring field theory (SSFT) $[55,56,57]$ where analogous conjectures exist $[58,59,60,61,62,63]$ and some have already been tested using the level truncation scheme [64, 65, 66, 67]. It is again natural to conjecture that at the tachyon vacuum the kinetic term does not involve any matter sector operator.

The paper is organised as follows. In section 2 we discuss the properties that we expect of the open string field theory action expanded around the tachyon vacuum, and construct examples of actions satisfying these properties. In section 3 we show how, starting from this action, and assuming the existence of a space-time independent solution of the equations of motion describing the original D-brane, we can calculate the ratio of tensions of the lump solutions of different dimensions. In the concluding section 4 we discuss various issues which are important for further extension of our analysis. These include a study of the uniqueness of the proposed action, the possibility of using level truncation scheme for calculating the overall normalization of the lump tension, an analysis of gauge fixing, generalization to superstrings, and a discussion on closed string states.

\section{String Field Theory Around the Tachyon Vac- uum}

Here we begin by considering open string field theory formulated on the background of a specific D-brane, focusing on its algebraic structure both before and after tachyon condensation to the vacuum where the brane disappears. After a discussion of the properties expected from the kinetic term of the string field theory around the tachyon vacuum we propose an ansatz consistent with such expectations.

as opposed to the string field tachyon condensate, the string field taking the original Dbrane configuration to the one with vanishing kinetic term is annihilated by the BRST operator. Thus the purely cubic action is unlikely to represent the tachyon vacuum. 


\subsection{Algebraic structure before and after condensation}

We begin with the cubic string field theory action describing the worldvolume theory of a $\mathrm{D}-(N-1)$-brane:

$$
S(\Phi)=-\frac{1}{g_{o}^{2}}\left[\frac{1}{2}\left\langle\Phi, Q_{B} \Phi\right\rangle+\frac{1}{3}\langle\Phi, \Phi * \Phi\rangle\right],
$$

where $g_{o}$ is the open string coupling constant and $\Phi$ is the open string field, conventionally taken to be Grassmann odd and of ghost number one for the classical action. In addition, $Q_{B}$ is the BRST operator, $\langle\cdot, \cdot\rangle$ is a bilinear inner product based on $\mathrm{BPZ}$ conjugation and $*$ denotes star-multiplication of string fields. The consistency of this classical action is guaranteed by the following identities involving the BRST operator

$$
\begin{aligned}
& Q_{B}^{2}=0, \\
& Q_{B}(A * B)=\left(Q_{B} A\right) * B+(-1)^{A} A *\left(Q_{B} B\right), \\
& \left\langle Q_{B} A, B\right\rangle=-(-)^{A}\left\langle A, Q_{B} B\right\rangle,
\end{aligned}
$$

and identities involving the inner product and the star operation

$$
\begin{aligned}
& \langle A, B\rangle=(-)^{A B}\langle B, A\rangle \\
& \langle A, B * C\rangle=\langle A * B, C\rangle \\
& A *(B * C)=(A * B) * C .
\end{aligned}
$$

In the sign factors, the exponents $A, B, \cdots$ denote the Grassmanality of the state, and should be read as $(-)^{A} \equiv(-)^{\epsilon(A)}$ where $\epsilon(A)=0(\bmod 2)$ for $A$ Grassmann even, and $\epsilon(A)=1(\bmod 2)$ for $A$ Grassmann odd. We also have:

$$
\begin{aligned}
& \epsilon(A * B)=\epsilon(A)+\epsilon(B) \\
& \operatorname{gh}(A * B)=\operatorname{gh}(A)+\operatorname{gh}(B),
\end{aligned}
$$

where gh denotes ghost number, and we take the ghost number of the $\mathrm{SL}(2, \mathrm{R})$ vacuum to be zero. Equations (2.2) and (2.3) guarantee that the above action is invariant under the gauge transformations:

$$
\delta \Phi=Q_{B} \Lambda+\Phi * \Lambda-\Lambda * \Phi,
$$

for any Grassmann-even ghost-number zero state $\Lambda$.

Let $\Phi_{0}$ be the string field configuration describing the tachyon vacuum, a solution of the classical field equations following from the action in (2.1):

$$
Q_{B} \Phi_{0}+\Phi_{0} * \Phi_{0}=0 .
$$


If $\widetilde{\Phi}=\Phi-\Phi_{0}$ denotes the shifted open string field, then the cubic string field theory action expanded around the tachyon vacuum has the form:

$$
S\left(\Phi_{0}+\widetilde{\Phi}\right)=S\left(\Phi_{0}\right)-\frac{1}{g_{o}^{2}}\left[\frac{1}{2}\langle\widetilde{\Phi}, Q \widetilde{\Phi}\rangle+\frac{1}{3}\langle\widetilde{\Phi}, \widetilde{\Phi} * \widetilde{\Phi}\rangle\right]
$$

Here $S\left(\Phi_{0}\right)$ is a constant, which according to the energetics part of the tachyon conjectures equals the mass $M$ of the D-brane when the D-brane extends over a space-time of finite volume. ${ }^{6}$ Indeed, the potential energy $V\left(\Phi_{0}\right)=-S\left(\Phi_{0}\right)$ associated to this string field configuration should equal minus the mass of the brane. The kinetic operator $Q$ is given in terms of $Q_{B}$ and $\Phi_{0}$ as:

$$
Q \widetilde{\Phi}=Q_{B} \widetilde{\Phi}+\Phi_{0} * \widetilde{\Phi}+\widetilde{\Phi} * \Phi_{0}
$$

More generally, on arbitrary string fields one would define

$$
Q A=Q_{B} A+\Phi_{0} * A-(-1)^{A} A * \Phi_{0} .
$$

The consistency of the action (2.7) is guaranteed from the consistency of the one in (2.1). Since neither the inner product nor the star multiplication have changed, the identities in (2.3) still hold. One can readily check that the identities in (2.2) hold when $Q_{B}$ is replaced by $Q$ [41]. Just as (2.1) is invariant under the gauge trasformations (2.5), the action in (2.7) is invariant under $\delta \widetilde{\Phi}=Q \Lambda+\widetilde{\Phi} * \Lambda-\Lambda * \widetilde{\Phi}$ for any Grassmann-even ghost-number zero state $\Lambda$.

Since the energy density of the brane represents a positive cosmological constant, it is natural to add the constant $-M=-S\left(\Phi_{0}\right)$ to (2.1). This will cancel the $S\left(\Phi_{0}\right)$ term in (2.7), and will make manifest the expected zero energy density in the final vacuum without D-brane. For the analysis around this final vacuum it suffices therefore to study the action

$$
S_{0}(\widetilde{\Phi}) \equiv-\frac{1}{g_{o}^{2}}\left[\frac{1}{2}\langle\widetilde{\Phi}, Q \widetilde{\Phi}\rangle+\frac{1}{3}\langle\widetilde{\Phi}, \widetilde{\Phi} * \widetilde{\Phi}\rangle\right] .
$$

\subsection{An ansatz for the SFT after condensation}

If we had a closed form solution $\Phi_{0}$ available, the problem of formulating SFT around the tachyon vacuum would be significantly simplified, as we would only have to understand the properties of the new kinetic operator $Q$ in

\footnotetext{
${ }^{6}$ As suggested in [10], we shall assume that the time interval has unit length so that the action can be identified with the negative of the potential energy for static configurations.
} 
(2.9). In particular we would like to confirm that its cohomology vanishes in accordance with the expectation that all conventional open string excitations disappear in the tachyon vacuum. Even the numerical approximations for $\Phi_{0}$ may give some indication if this is the case $[9,17,18]$.

Previous experience with background deformations (small and large) in SFT indicates that even if we knew $\Phi_{0}$ explicitly and constructed $S_{0}(\widetilde{\Phi})$ using eq.(2.10), this may not be the most convenient form of the action. Typically a nontrivial field redefinition is necessary to bring the shifted SFT action to the canonical form representing the new background [73]. In fact, in some cases, such as in the formulation of open SFT for D-branes with various values of magnetic fields, it is simple to formulate the various SFT's directly [74], but the nontrivial classical solution relating theories with different magnetic fields are not known. This suggests that if a simple form exists for the SFT action around the tachyon vacuum it might be easier to guess it than to derive it.

In proposing a simple form of the tachyon action, we have in mind field redefinitions of the action in (2.10) that leave the cubic term invariant but simplify the operator $Q$ in (2.9) by transforming it into a simpler operator $\mathcal{Q}$. To this end we consider homogeneous field redefinitions of the type

$$
\widetilde{\Phi}=e^{K} \Psi,
$$

where $K$ is a ghost number zero Grassmann even operator. In addition, we require

$$
\begin{aligned}
& K(A * B)=(K A) * B+A *(K B), \\
& \langle K A, B\rangle=-\langle A, K B\rangle .
\end{aligned}
$$

These properties guarantee that the form of the cubic term is unchanged and that after the field redefinition the action takes the form

$$
\mathcal{S}(\Psi) \equiv-\frac{1}{g_{0}^{2}}\left[\frac{1}{2}\langle\Psi, \mathcal{Q} \Psi\rangle+\frac{1}{3}\langle\Psi, \Psi * \Psi\rangle\right]
$$

where

$$
\mathcal{Q}=e^{-K} Q e^{K}
$$

Again, gauge invariance only requires:

$$
\begin{aligned}
& \mathcal{Q}^{2}=0, \\
& \mathcal{Q}(A * B)=(\mathcal{Q} A) * B+(-1)^{A} A *(\mathcal{Q} B), \\
& \langle\mathcal{Q} A, B\rangle=-(-)^{A}\langle A, \mathcal{Q} B\rangle .
\end{aligned}
$$


These identities hold by virtue of (2.12) and (2.14). We will proceed here postulating a $\mathcal{Q}$ that satisfies these identities as well as other conditions, since lacking knowledge of $\Phi_{0}$ the above field redefinitions cannot be attempted.

The choice of $\mathcal{Q}$ will be required to satisfy the following properties:

- The operator $\mathcal{Q}$ must be of ghost number one and must satisfy the conditions (2.15) that guarantee gauge invariance of the string action.

- The operator $\mathcal{Q}$ must have vanishing cohomology.

- The operator $\mathcal{Q}$ must be universal, namely, it must be possible to write without reference to the brane boundary conformal field theory.

The simplest possibility would be to set $\mathcal{Q}=0$. This would result in the purely cubic version of open string field theory [50]. Indeed, it has long been tempting to identify the tachyon vacuum with a theory where the kinetic operator vanishes, especially because lacking the kinetic term the string field gauge symmetries are not spontaneously broken. Nevertheless, there are well-known complications with this identification. At least in the construction of [50] the string field shift $\bar{\Phi}$ relating the cubic to the purely cubic SFT's involves the subtle identity operator $\mathcal{I}$ and appears to satisfy $Q \bar{\Phi}=0$ as well as $\bar{\Phi} * \bar{\Phi}=0$. The tachyon condensate definitely does not satisfy these two identities. We therefore search for nonzero $\mathcal{Q}$.

We can satisfy the three requirements by letting $\mathcal{Q}$ be constructed purely from ghost operators. In particular we claim that the ghost number one operators

$$
\mathcal{C}_{n} \equiv c_{n}+(-)^{n} c_{-n}, \quad n=0,1,2, \cdots
$$

satisfy the properties

$$
\begin{aligned}
& \mathcal{C}_{n} \mathcal{C}_{n}=0 \\
& \mathcal{C}_{n}(A * B)=\left(\mathcal{C}_{n} A\right) * B+(-1)^{A} A *\left(\mathcal{C}_{n} B\right) \\
& \left\langle\mathcal{C}_{n} A, B\right\rangle=-(-)^{A}\left\langle A, \mathcal{C}_{n} B\right\rangle
\end{aligned}
$$

The first property is manifest. The last property follows because under BPZ conjugation $c_{n} \rightarrow(-)^{n+1} c_{-n}$. The second property follows from the conservation law

$$
\left\langle V_{3}\right|\left(\mathcal{C}_{n}^{(1)}+\mathcal{C}_{n}^{(2)}+\mathcal{C}_{n}^{(3)}\right)=0,
$$


on the three string vertex [15]. These conservation laws arise from consideration of integrals of the form $\int d z c(z) \varphi(z)$ where $\varphi(z)(d z)^{2}$ is a globally defined quadratic differential. In fact, the conservation law for $\mathcal{C}_{0}$ arises from a familiar Jenkins-Strebel quadratic differential with a second order pole at each of the three puntures. This quadratic differential, in fact, defines the geometry of the vertex. Its horizontal trajectories show the open strings and their interaction.

Each of the operators $\mathcal{C}_{n}$ has vanishing cohomology since for each $n$ the operator $B_{n}=\frac{1}{2}\left(b_{n}+(-)^{n} b_{-n}\right)$ satisfies $\left\{\mathcal{C}_{n}, B_{n}\right\}=1$. It then follows that whenever $\mathcal{C}_{n} \psi=0$, we have $\psi=\left\{\mathcal{C}_{n}, B_{n}\right\} \psi=\mathcal{C}_{n}\left(B_{n} \psi\right)$, showing that $\psi$ is $\mathcal{C}_{n}$ trivial. Finally, since they are built from ghost oscillators, all $\mathcal{C}_{n}$ 's are manifestly universal.

It is clear from the structure of the conditions (2.15) that they are satisfied for the general choice:

$$
\mathcal{Q}=\sum_{n=0}^{\infty} a_{n} \mathcal{C}_{n}
$$

where the $a_{n}$ 's are constant coefficients. We will discuss in section 4.1 how different choices for these constants result in kinetic operators that are sometimes related by field redefinitions.

A subset of the above kinetic operators were considered by Horowitz et.al.[49], as they discussed how the purely cubic SFT could have solutions leading to forms different from the conventional cubic SFT. In particular they noticed that operators built just with ghost oscillators could appear as the kinetic term of an action obtained by shifting by a (formal) solution of the cubic theory.

There may be other choices of $\mathcal{Q}$ satisfying all the requirements stated above. Fortunately, our analysis of section 3 will not require the knowledge of the detailed form of $\mathcal{Q}$, as long as it does not involve any matter operators. To this end, it will be useful to note that since $\mathcal{Q}$ does not involve matter operators, we can fix the gauge by choosing a gauge fixing condition that also does not involve any matter operator. In such a gauge, the propagator will factor into a non-trivial operator in the ghost sector, and the identity operator in the matter sector. Some details of gauge fixing are discussed in section (4.3). 


\section{The Lump Solutions and Their Tensions}

In this section we shall analyze classical solutions of the string field action (2.13) introduced in the previous section. The analysis will proceed in three steps: 1) analysis of the tachyon effective action, 2) construction of the lump solution, and 3) computation of the ratio of the tensions of the lump solutions.

\subsection{Analysis of the tachyon effective action}

We shall consider a situation where we start with an unstable D- $(N-1)$ brane of the bosonic string theory, and consider the string field theory action expanded around the tachyon vacuum of this theory. According to our postulates of the previous section this action will have the form given in eq.(2.13). Given this action and a suitable gauge fixing which does not involve any matter operator, the propagator involves purely ghost sector operators. Since both the vertex and the propagator now factorize into matter sector contribution and ghost sector contribution, a general $N$-point function will also factorize into a matter sector contribution and a ghost sector contribution.

We shall now concentrate on the $n$-tachyon Green's function. If we denote by $J(p)$ the current coupling to a tachyon of momentum $(-p)$, then the generating functional of the tachyon Green's functions has the form:

$$
W[J]=\sum_{n=2}^{\infty} \frac{1}{n !} \int d^{N} p_{1} \ldots d^{N} p_{n} g^{(n)}\left(p_{1}, \ldots p_{n}\right) J\left(p_{1}\right) \ldots J\left(p_{n}\right) \delta\left(\sum_{i=1}^{n} p_{i}\right) .
$$

Here $g^{(n)}\left(p_{1}, \ldots p_{n}\right) \delta\left(\sum_{i=1}^{n} p_{i}\right)$ is the $n$-tachyon off-shell amplitude obtained by adding all tree level connected Feynman diagrams. Note that since we consider a propagator that involves just simple ghost factor, its world sheet interpretation is that of gluing with a zero length strip and inserting a ghost operator. Thus the propagator collapses to an overlap with a ghost insertion. ${ }^{7}$ Recall that the cubic vertex corresponds to the symmetric gluing of three semi-infinite strips across the open edges, half edges at a time. It follows that for $n$ external tachyons any connected Feynman graph constructed with this vertex and the overlap propagator will simply be built by the symmetric gluing of $n$-semiinfinite strips across their open edges, half edges at

\footnotetext{
${ }^{7}$ If the ghost kinetic operator does not have a simple inverse the fact remains that in the matter sector the propagator will act as an overlap.
} 
a time. Mapping this world sheet into a unit disk, each strip turns into a wedge of angle $2 \pi / n$. Except for the ghost, insertions this can be viewed as the iterated star product of $n$ tachyon vertex operators. In fact, such iterated products are precisely the ones used in the WZW-like superstring field theory [55] as discussed in detail in [65].

It follows from the above remarks that the $n$-tachyon off shell amplitude can be written as the following correlation function on a unit disk:

$$
g^{(n)}\left(p_{1}, \ldots p_{n}\right) \delta\left(\sum_{i=1}^{n} p_{i}\right)=C_{n}\left\langle\prod_{k=1}^{n}\left(f_{k}^{(n)} \circ e^{i p_{k} \cdot X(0)}\right)\right\rangle
$$

where $\langle\cdots\rangle$ denotes matter sector correlation function, $C_{n}$ is a constant representing contribution from the ghost sector correlator, $f_{k}^{(n)} \circ e^{i p_{k} \cdot X(0)}$ denotes the conformal transform of $e^{i p_{k} \cdot X(0)}$ by the function $f_{k}^{(n)}(z)$ :

$$
f_{k}^{(n)} \circ e^{i p_{k} \cdot X(0)}=\left|f_{k}^{(n) \prime}(0)\right|^{p_{k}^{2}} e^{i p_{k} \cdot X\left(f_{k}^{(n)}(0)\right)},
$$

and [65]:

$$
f_{k}^{(n)}(z)=e^{2 \pi i(k-1) / n}\left(\frac{1+i z}{1-i z}\right)^{2 / n}
$$

Using the relations:

$$
f_{k}^{(n)}(0)=e^{2 \pi i(k-1) / n}, \quad f_{k}^{(n) \prime}(0)=e^{2 \pi i(k-1) / n} \frac{4}{n} i
$$

and

$$
\left\langle\prod_{k=1}^{n} e^{i p_{k} \cdot X\left(f_{k}^{(n)}(0)\right)}\right\rangle=\delta\left(\sum_{k=1}^{n} p_{k}\right) \prod_{k \neq l}\left|f_{k}^{(n)}(0)-f_{l}^{(n)}(0)\right|^{p_{k} \cdot p_{l}},
$$

we get

$$
g^{(n)}\left(p_{1}, \ldots p_{n}\right)=C_{n} \exp \left[\left(\ln \frac{4}{n}\right) \sum_{k=1}^{n} p_{k}^{2}+\sum_{\substack{k, l=1 \\ k \neq l}}^{n} p_{k} \cdot p_{l} \ln \left(2 \sin \left(\frac{\pi}{n}|k-l|\right)\right)\right]
$$

While $g^{(n)}$ given above is cyclically symmetric in $p_{1}, \ldots p_{n}$, only the fully symmetric part of $g^{(n)}$ contributes to $W$. The tachyon propagator $g^{(2)}$ is a momentum independent constant.

We now perform a series of manipulations familiar in path integral quantization of field theories. The tachyon effective action can be obtained from 
the generating functional $W$ by taking the Legendre transform. As usual, we introduce the classical field expectation value as

$$
\phi(q)=\phi_{c}[q, J]
$$

where

$$
\begin{aligned}
& \phi_{c}\left[-p_{1}, J\right] \equiv \frac{\delta W}{\delta J\left(p_{1}\right)} \\
&= \sum_{n=2}^{\infty} \frac{1}{(n-1) !} \int d^{N} p_{2} \ldots d^{N} p_{n} g^{(n)}\left(p_{1}, \ldots p_{n}\right) \\
& \cdot \delta\left(\sum_{i=1}^{n} p_{i}\right) J\left(p_{2}\right) \ldots J\left(p_{n}\right) .
\end{aligned}
$$

Note that $\phi_{c}[q, J]$ is a function of the momentum variable $q$ and a functional of $J(p)$. In deriving the above equation we have used the cyclic symmetry of $g^{(n)}$. The effective action, a functional of classical fields $\phi(q)$, is given by,

$$
\Gamma[\phi]=\int d^{N} p J(p) \phi(-p)-W[J],
$$

where $J$ above is the source that gives rise to $\phi(q)$ through (3.8), (3.9). Since $g^{(2)}$ is momentum independent, the coefficient of the quadratic term in $\Gamma[\phi]$ will be momentum independent and the higher order terms will have exponential dependence on the momenta. This resembles the action of the $p$-adic string theory expanded around the tachyon vacuum[68, 35] after a momentum dependent rescaling of the tachyon field. As in $p$-adic string theory we shall see that gaussians play an important role in constructing lump solutions.

It follows from equation (3.10) that

$$
J(-p)=\frac{\delta \Gamma[\phi]}{\delta \phi(p)} .
$$

Thus, a solution to the equations of motion arising from setting the variation of the effective action to zero amounts to setting the current $J$ to zero. Therefore, classical solutions are of the form:

$$
\phi(q)=\phi_{c}[q, J(p)=0] .
$$

Since $\phi_{c}[q, J]$ has a Taylor series expension in $J$ starting with linear power, this would seem to always give the trivial solution $\phi(q)=0$. However, $W$ and $\phi_{c}$ have branch points in the complex $[J(p)]$ space, and by going 
around these branch points, one may get $\phi_{c}[q, J(p)=0] \neq 0$. Every such path in the $J$ space will give rise to a valid solution of the equations of motion. ${ }^{8}$

\subsection{Setup for lump solutions}

We shall begin by examining translationally invariant solutions of the equations of motion. (The original D- $(N-1)$-brane configuration will be represented by such a solution.) In momentum space a translationally invariant solution $\phi(p)$ is proportional to $\delta(p)$ where $p$ is an $N$-dimensional momentum vector. Consider eq. (3.9) with a delta function source $J(p)=u \delta(p)$. We find

$$
\phi_{c}[q, u \delta(p)] \equiv F(u) \delta(q)
$$

with

$$
F(u)=\sum_{n=2}^{\infty} \frac{1}{(n-1) !} u^{n-1} g^{(n)}(0, \ldots 0)=\sum_{n=2}^{\infty} \frac{1}{(n-1) !} C_{n} u^{n-1},
$$

where use was made of (3.7) for zero momentum. Now suppose that the function $F(u)$ has a branch point $u_{b}$ in the complex $u$ plane such that as $u$ returns to 0 after going around this branch point, $F(u)$ returns to $\phi_{0}$. Thus in this branch

$$
\lim _{u \rightarrow 0^{\prime}} F(u)=\phi_{0},
$$

where $0^{\prime}$ is used to denote the origin on this branch. Hence $\lim _{u \rightarrow 0^{\prime}} \phi_{c}(q, u \delta(p))$ $=\phi_{0} \delta(q)$. Eq. (3.12) then implies the existence of a translationally invariant solution of the equations of motion of the form

$$
\phi(p)=\phi_{0} \delta(p) .
$$

Conversely, existence of a solution of the form given in (3.16) implies that the function $F(u)$ has a branch point $u_{b}$ in the complex $u$ plane such that as $u$ returns to 0 after going around the branch point, $F(u)$ returns to $\phi_{0}$. We

\footnotetext{
${ }^{8}$ The situation can be illustrated by considering the zero momentum sector of the $\phi^{3}$ field theory. In this case, at the tree level, $\Gamma[\phi]=-\frac{1}{2} m^{2} \phi^{2}+\frac{1}{3} \lambda \phi^{3}$. This gives $J=-m^{2} \phi+\lambda \phi^{2}$, and $\phi=\frac{1}{2 \lambda}\left(m^{2} \pm \sqrt{m^{4}+4 \lambda J}\right) \equiv \phi_{c}[J]$. If we choose the - sign in front of the square root, $\phi_{c}[J]$ vanishes at $J=0$ and has a Taylor series expansion in $J$ starting at linear order. But going around the branch point at $J=-m^{4} /(4 \lambda)$ in the complex $J$ plane, we can move to the other branch with + sign in front of the square root. Now if we return to the origin $J=0$, we get $\phi_{c}[0]=m^{2} / \lambda$. This is indeed the location of an extremum of $\Gamma\left[\phi_{c}\right]$ and hence represents a solution of the equations of motion.
} 
shall choose $\phi_{0}$ to be the value that describes the original D-brane solution before tachyon condensation; such a solution exists by assumption. In what follows, we shall assume that the branch point $u_{b}$ is the closest singularity to the origin in the complex $u$ plane, so that the growth of the coefficients in the Taylor series expansion of $F(u)$ is controlled by $u_{b}$. In this case, the radius of convergence of the Taylor series expansion of $F(u)$ given in eq.(3.14) is $\left|u_{b}\right|$.

Now suppose we find a pair of functions $\psi(p)$ and $\chi(p)$ such that,

$$
H(u, q) \equiv \phi_{c}[q, u \psi(p)]-F(u) \chi(q)
$$

has the property that the rate of growth of the coefficients in the Taylor series expansion of $H(u, q)$ in $u$ is slower than that of $F(u) \chi(q)$. In this case we can conclude that

1. $H(u, q)$ does not have a singularity for $|u|<\left|u_{b}\right|$.

2. $H(u, q)$ either has no singularity at $u=u_{b}$ or its singularities at $u=u_{b}$ are non-leading compared to that of $F(u) \chi(q)$.

We can now consider tracing the path around $u_{b}$ in the complex $u$-plane so that $F(u)$ approaches $\phi_{0}$ as $u$ returns to the origin. If $H(u, q)$ has no singularity at $u=u_{b}$, then it will return to zero. Otherwise it will return to some function $h(q)$

$$
\lim _{u \rightarrow 0^{\prime}} H(u, q)=h(q) .
$$

As a result, on this branch (3.17) gives:

$$
\lim _{u \rightarrow 0^{\prime}} \phi_{c}[q, u \psi(p)]=\phi_{0} \chi(q)+h(q) .
$$

Eq.(3.12) then implies that

$$
\phi(q)=\phi_{0} \chi(q)+h(q),
$$

is a solution of the equations of motion. Thus given two functions $\psi(q)$ and $\chi(q)$ satisfying the property stated below eq.(3.17), and a translationally invariant solution $\phi_{0} \delta(q)$, we can find a space-time dependent solution $\phi_{0} \chi(q)+h(q)$. Note that we have defined $\chi(q)$ to be such that $F(u) \chi(q)$ is the leading contribution to $\phi_{c}(q, u \psi(p))$; so by definition, the left-over piece $H$ is sub-leading i.e. its power series expansion should converge faster than that of $F(u) \chi(q)$. However, the existence of such a $\chi(q)$ is not guaranteed 
a priori since the $u$-dependence of $\phi_{c}(q, u \psi(p))$ could be very different from that of $\phi_{c}(q, u \delta(p))$.

We shall now make the convergence condition precise. Let us define

$$
R_{n}\left(-p_{1}\right) \equiv \frac{\int d^{N} p_{2} \ldots d^{N} p_{n} g^{(n)}\left(p_{1}, \ldots p_{n}\right) \delta\left(\sum_{i=1}^{n} p_{i}\right) \psi\left(p_{2}\right) \ldots \psi\left(p_{n}\right)}{g^{(n)}(0, \ldots 0) \chi\left(-p_{1}\right)} .
$$

Making use of this definition, and of eqs. (3.9), (3.14) and (3.21), equation (3.17) gives

$$
H(u,-p)=\chi(-p) \sum_{n=2}^{\infty} \frac{u^{n-1}}{(n-1) !} C_{n}\left[R_{n}(-p)-1\right] .
$$

We need to ensure that the Taylor series expansion of $H(u, q)$ converges more rapidly than the Taylor series expansion of $F(u) \chi(q)$ given in eq.(3.14). This, in turn requires that

$$
\lim _{n \rightarrow \infty} R_{n}(-p)=1
$$

Note that the dependence on the constants $C_{n}$ arising from ghost correlators in (3.7) drops out for $R_{n}$ defined in eq.(3.21). Equation (3.23) will be the key equation in the analysis that follows.

\subsection{Equations for lump solutions}

In order to proceed further, we need to have an ansatz for $\psi(p)$ and $\chi(p)$. We use the following ansatz:

$$
\psi(p)=K \exp \left(-\alpha p_{\perp}^{2} / 2\right) \delta\left(p_{\|}\right), \quad \chi(p)=\gamma K \exp \left(-\beta p_{\perp}^{2} / 2\right) \delta\left(p_{\|}\right),
$$

where $K, \alpha, \beta$ and $\gamma$ are constants to be determined, and $\left(p_{\|}, p_{\perp}\right)$ denotes a decomposition of the vector $p$ into two orthogonal subspaces of dimensions $N_{\perp}$ and $N_{\|}$respectively $\left(N_{\perp}+N_{\|}=N\right)$. In position space labelled by $\left(x_{\|}, x_{\perp}\right)$ this corresponds to a configuration which is independent of $x_{\|}$and has the form of a gaussian along $x_{\perp}$. Thus a natural interpretation of this solution is that of a solitonic $\left(N_{\|}-1\right)$ brane along $x_{\|}$. Substituting (3.24) into eq.(3.21), we get

$$
\begin{aligned}
& \gamma K R_{n}\left(-p_{1}\right) g^{(n)}(0, \ldots 0) \exp \left(-\beta p_{1 \perp}^{2} / 2\right) \delta\left(p_{1 \|}\right) \\
& =K^{n-1} \int d^{N} p_{2} \ldots d^{N} p_{n} g^{(n)}\left(p_{1}, \ldots p_{n}\right) \\
& \cdot \delta\left(\sum_{i=1}^{n} p_{i}\right) \exp \left(-\frac{\alpha}{2} \sum_{i=2}^{n} p_{i \perp}^{2}\right) \prod_{i=2}^{n} \delta\left(p_{i \|}\right) .
\end{aligned}
$$


Using (3.7) in eq.(3.25), performing the integrals over $p_{i \|}$, with $i=2, \cdots n$, and using the freedom of setting $p_{1 \|}=0$ in $g^{(n)}\left(p_{1}, \ldots p_{n}\right)$ and $R_{n}\left(-p_{1}\right)$ due to the accompanying factors of $\delta\left(p_{1 \|}\right)$ we get

$$
\begin{gathered}
\gamma R_{n}\left(-p_{1 \perp}\right) \exp \left(-\frac{(\alpha+\beta)}{2} p_{1 \perp}^{2}\right) \\
=K^{n-2} \int d^{N_{\perp}} p_{2 \perp} \ldots d^{N_{\perp}} p_{n \perp} \delta\left(\sum_{i=1}^{n} p_{i \perp}\right) \exp \left(\sum_{k, l=1}^{n} a_{k-l} p_{k \perp} \cdot p_{l \perp}\right),(3.2
\end{gathered}
$$

where

$$
\begin{aligned}
& a_{0}=\ln \frac{4}{n}-\frac{\alpha}{2} \\
& a_{k}=\ln \left|2 \sin \left(\frac{\pi k}{n}\right)\right| \text { for } k \neq 0 .
\end{aligned}
$$

We now multiply both sides of the equation by $e^{i p_{1 \perp} \cdot x_{\perp}}$ for some $N_{\perp}$ dimensional vector $x_{\perp}$ and integrate over $p_{1 \perp}$. Demanding that in the $n \rightarrow \infty$ limit $R_{n}$ should approach 1 , we get

$$
\begin{gathered}
\gamma\left(\frac{2 \pi}{\alpha+\beta}\right)^{N_{\perp} / 2} \exp \left(-\frac{x_{\perp}^{2}}{2(\alpha+\beta)}\right) \\
=\lim _{n \rightarrow \infty}\left[K^{n-2} \int \prod_{k=1}^{n} d^{N_{\perp}} p_{k \perp} \delta\left(\sum_{i=1}^{n} p_{i \perp}\right) \exp \left(i p_{1 \perp} \cdot x_{\perp}+\sum_{k, l=1}^{n} a_{k-l} p_{k \perp} \cdot p_{l \perp}\right)\right] .
\end{gathered}
$$

In order to do the integral over $p_{i \perp}$ on the right hand side, we take help of the discrete Fourier transform. We introduce new variables $\phi_{l \perp}$ as follows:

$$
\phi_{l \perp}=\frac{1}{\sqrt{n}} \sum_{k=1}^{n} p_{k \perp} e^{-2 \pi i(k-1) l / n}, \quad 1 \leq l \leq n .
$$

Then

$$
p_{k \perp}=\frac{1}{\sqrt{n}} \sum_{l=1}^{n} \phi_{l \perp} e^{2 \pi i(k-1) l / n} .
$$

The reality condition on $\phi_{l \perp}$ is of the form:

$$
\phi_{l \perp}^{*}=\phi_{(n-l) \perp} \quad \text { for } \quad 1 \leq l \leq(n-1), \quad \phi_{n \perp}^{*}=\phi_{n \perp} .
$$

Let us first take $n$ to be odd. In that case we can introduce a new set of variables $\xi_{s \perp}, \eta_{s \perp}$ through the relations:

$$
\phi_{s \perp}=\frac{1}{\sqrt{2}}\left(\xi_{s \perp}+i \eta_{s \perp}\right) \quad \text { for } \quad 1 \leq s \leq \frac{n-1}{2} .
$$


We take $\phi_{n \perp}, \xi_{s \perp}$ and $\eta_{s \perp}$ as independent variables, and express the right hand side of eq.(3.28) in terms of these variables. This gives

$$
\begin{aligned}
& \gamma\left(\frac{2 \pi}{\alpha+\beta}\right)^{N_{\perp} / 2} \exp \left(-\frac{x_{\perp}^{2}}{2(\alpha+\beta)}\right) \\
= & \lim _{n \rightarrow \infty}\left[K^{n-2} \int d^{N_{\perp}} \phi_{n \perp} \prod_{s=1}^{(n-1) / 2}\left(d^{N_{\perp}} \xi_{s \perp} d^{N_{\perp}} \eta_{s \perp}\right) \delta\left(\sqrt{n} \phi_{n \perp}\right)\right. \\
& \left.\exp \left(\frac{i}{\sqrt{n}}\left(\phi_{n \perp}+\sqrt{2} \sum_{s=1}^{(n-1) / 2} \xi_{s \perp}\right) \cdot x_{\perp}-b_{n} \phi_{n \perp}^{2}-\sum_{s=1}^{(n-1) / 2} b_{s}\left(\xi_{s \perp}^{2}+\eta_{s \perp}^{2}\right)\right)\right],
\end{aligned}
$$

where

$$
b_{s}=-\sum_{k=0}^{n-1} a_{k} \cos \left(\frac{2 \pi k s}{n}\right)=\ln \frac{n}{4}+\frac{\alpha}{2}-\sum_{k=1}^{n-1} \ln \left(2 \sin \left(\frac{\pi k}{n}\right)\right) \cos \left(\frac{2 \pi k s}{n}\right) .
$$

We can now perform the integrals over $\phi_{n \perp}, \xi_{s \perp}$ and $\eta_{s \perp}$ explicitly. This gives

$$
\begin{aligned}
& \gamma\left(\frac{2 \pi}{\alpha+\beta}\right)^{N_{\perp} / 2} \exp \left(-\frac{x_{\perp}^{2}}{2(\alpha+\beta)}\right) \\
= & \lim _{n \rightarrow \infty}\left[K^{n-2}\left\{\frac{1}{\sqrt{n}}\left(\prod_{s=1}^{(n-1) / 2} \frac{\pi}{b_{s}}\right)\right\}^{N_{\perp}} \exp \left(-\frac{1}{2 n} x_{\perp}^{2} \sum_{s=1}^{(n-1) / 2} \frac{1}{b_{s}}\right)\right] .
\end{aligned}
$$

Using the symmetry $b_{s}=b_{n-s}$, we can rewrite this equation as

$$
\begin{aligned}
& \gamma\left(\frac{2 \pi}{\alpha+\beta}\right)^{N_{\perp} / 2} \exp \left(-\frac{x_{\perp}^{2}}{2(\alpha+\beta)}\right) \\
= & \lim _{n \rightarrow \infty}\left[K^{n-2}\left\{\frac{1}{n}\left(\prod_{s=1}^{n-1} \frac{\pi}{b_{s}}\right)\right\}^{N_{\perp} / 2} \exp \left(-\frac{1}{4 n} x_{\perp}^{2} \sum_{s=1}^{n-1} \frac{1}{b_{s}}\right)\right] .
\end{aligned}
$$

For $n$ even, we take the independent real variables to be $\phi_{n \perp}, \phi_{\frac{n}{2} \perp}, \xi_{s \perp}$ and $\eta_{s \perp}$ for $1 \leq s \leq(n-2) / 2$. Repeating the analysis, we arrive at exactly the same equation (3.36). Thus eq.(3.36) is valid irrespective of whether we take the limit $n \rightarrow \infty$ keeping $n$ even or odd. This is the main equation that must be analyzed. 


\subsection{Solving the lump equations}

We shall now try to examine if we can find solutions of (3.36) where $\alpha, \beta, \gamma$ and $K$ are finite. To this end let us define the functions:

$$
f(\alpha, n)=\sum_{s=1}^{n-1} \ln \frac{\pi}{b_{s}}-\ln n, \quad k(\alpha, n)=\frac{1}{n} \sum_{s=1}^{n-1} \frac{1}{b_{s}} .
$$

Comparing the two sides of equation (3.36) we find that

$$
\begin{gathered}
\beta=\lim _{n \rightarrow \infty} \frac{2}{k(\alpha, n)}-\alpha \equiv C(\alpha), \\
\lim _{n \rightarrow \infty}\left[(n-2) \ln K+\frac{1}{2} N_{\perp} f(\alpha, n)-\ln \gamma-\frac{1}{2} N_{\perp} \ln \frac{2 \pi}{\alpha+\beta}\right]=0 .
\end{gathered}
$$

Equation (3.38) arises from comparing the coefficients of the gaussians in $x_{\perp}$ in the two sides of equation (3.36). These coefficients must coincide. Once the gaussians coincide, the prefactors must coincide as well. Eqn. (3.39) arises by taking the logarithms of the prefactors in (3.36).

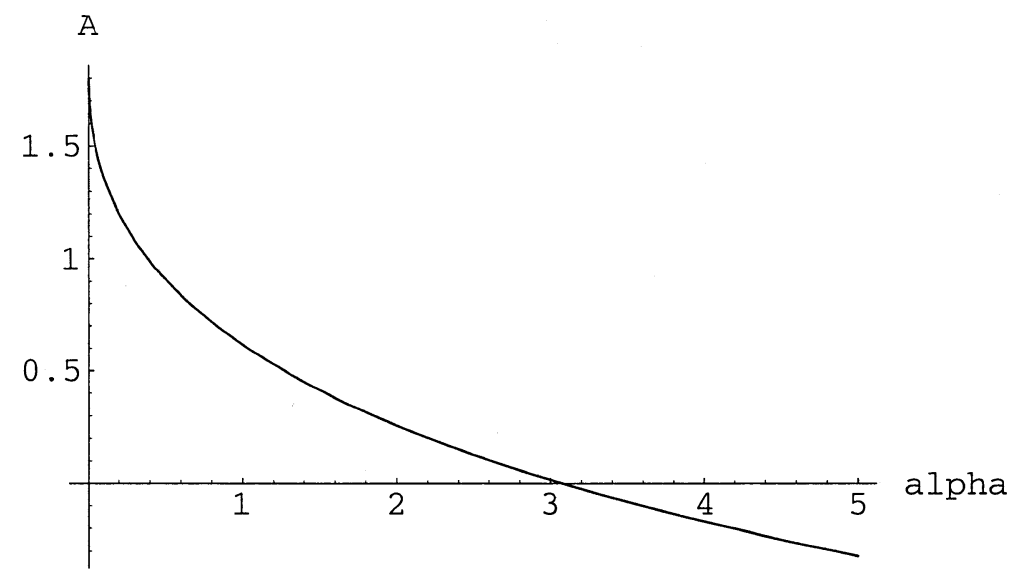

Figure 1: The function $A(\alpha)$ for $n=50$ (dashed) and $n=500$ (continuous). Actually the error is so small that the lines are essentially on top of each other.

Numerical analysis shows that the function $k(\alpha, n)$ defined through eq.(3.37) remains finite in the $n \rightarrow \infty$ limit. Hence $C(\alpha)$ is well defined. Furthermore, $f(\alpha, n)$ defined in eq. (3.37) has the form

$$
f(\alpha, n)=A(\alpha) n+B(\alpha)+s_{n}(\alpha),
$$




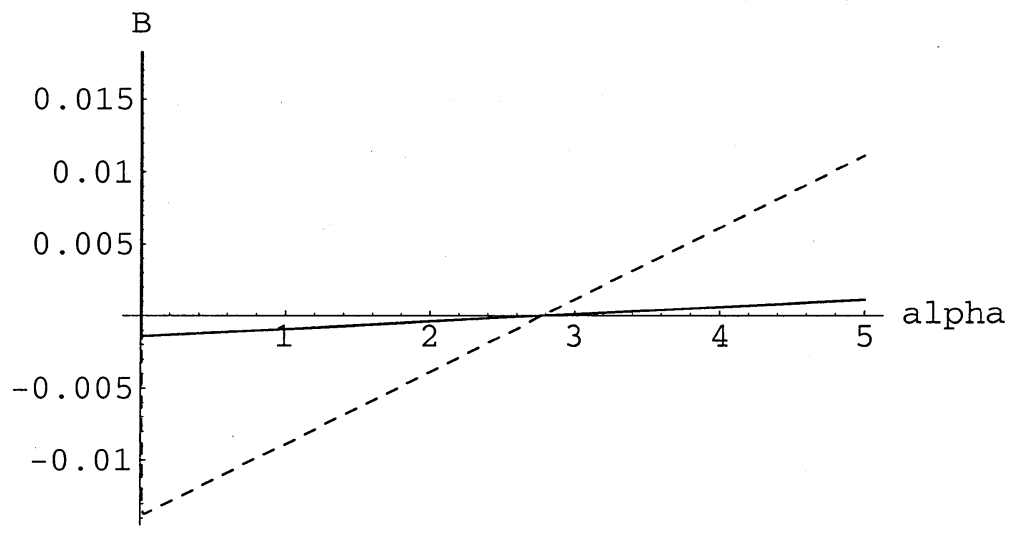

Figure 2: The function $\mathcal{B}(n, \alpha)$ (equation (3.41)) plotted for $n=50$ (dashed) and $n=500$ (continuous).

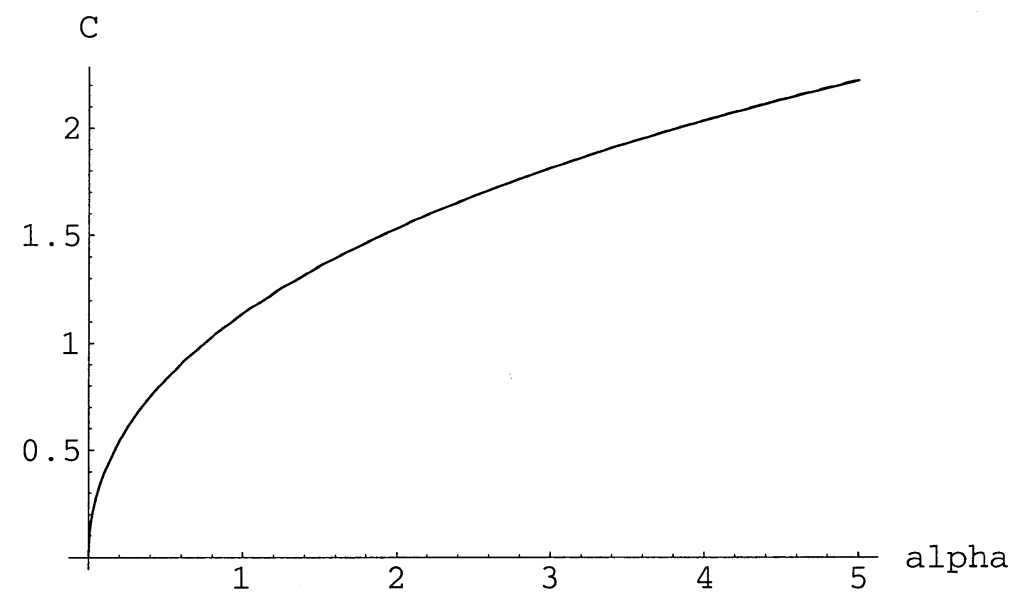

Figure 3: The function $C(\alpha)$ (eqn. (3.38)) for $n=50$ (dashed) and $n=500$ (continuous). Actually the error is so small that the lines are essentially on top of each other.

where $s_{n}(\alpha)$ vanishes in the $n \rightarrow \infty$ limit. In fact the function $B(\alpha)$ appears to vanish identically, but we shall for the time being, not explicitly set it to zero. We show in figure 1 a plot of $A(\alpha)$ calculated numerically as $f(\alpha, n+$ 1) $-f(\alpha, n)$ for $n=50$ (in dashed lines) and for $n=500$ (in continuous lines). Actually the error is so small that the lines are essentially on top of 
each other. In figure 2 we plot the quantity

$$
\mathcal{B}(n, \alpha) \equiv 2 f(\alpha, n)-f(\alpha, 2 n)=B(\alpha)+2 s_{n}(\alpha)-s_{2 n}(\alpha) .
$$

With $s_{n} \rightarrow 0$ as $n \rightarrow \infty$, for sufficiently large $n$ we have that $\mathcal{B}(n, \alpha)$ approaches $B(\alpha)$. Thus a nonzero $B(\alpha)$ would show as a constant function in the plot of $\mathcal{B}(n, \alpha)$. Nevertheless, the numerical evidence is that $\mathcal{B}(n, \alpha)$ goes to zero accurately as $n$ is made large. Indeed, as can be seen in figure 2 even for $n=50$ (dashed line) $\mathcal{B}(n, \alpha)$ is quite small over the considered range. When $n=500$ (continuous line) $\mathcal{B}(n, \alpha)$ becomes much smaller. We have explored this vanishing taking $n \sim 5000$ and have concluded that with very high accuracy (about one part in ten thousand)

$$
B(\alpha) \simeq 0,
$$

for the range of $\alpha$ shown in the figure. Having concluded that $B(\alpha)=0$, the graph of $\mathcal{B}(n, \alpha)$ gives us information about the $s_{n}(\alpha)$ terms. We have found that the leading $s_{n}(\alpha)$ term is of order $\mathcal{O}(1 / n)$. With $s_{n}(\alpha) \sim 1 / n$, and for large $n$ we have that $\mathcal{B}(n, \alpha) \simeq 3 s_{n}(\alpha) / 2$ (see (3.41)). Thus figure 2 is giving us a plot of the leading $s_{n}(\alpha)$ term. Note, that there is a value $\bar{\alpha}$ for which $\mathcal{B}(n, \bar{\alpha})=0$. We determined numerically this value to be $\bar{\alpha} \simeq 2.772588$ and believe that it corresponds exactly to $\bar{\alpha}=4 \ln (2)$. Moreover the dependence of $s_{n}(\alpha)$ on $\alpha$ seems linear to high accuracy for a large range around $\bar{\alpha}$ (see figure). We have determined that

$$
s_{n}(\alpha) \simeq \frac{1}{2} \frac{0.3333}{n}(\alpha-\bar{\alpha})+\mathcal{O}\left(1 / n^{2}\right), \quad \bar{\alpha}=4 \ln (2) .
$$

The coefficient 0.3333 may really take the exact value of $1 / 3$ but we do not know for certain. Our investigations also suggest that for $\alpha=\bar{\alpha}$ the $1 / n^{2}$ corrections vanish while the $1 / n^{3}$ corrections are nonzero. It is hoped that these observations may help develop an analytical approach to this large $n$ problem. Finally in figure 3 we show the function $C(\alpha)$. This concludes our discussion of the properties of (3.40).

Substituting (3.40) into (3.39) and comparing terms linear in $n$ as well as the constant terms in both sides of the equation we get: ${ }^{9}$

$$
\begin{aligned}
\ln K & =-\frac{1}{2} N_{\perp} A(\alpha), \\
\ln \gamma & =\frac{1}{2} N_{\perp}\left(-\ln \frac{2 \pi}{\alpha+\beta}+B(\alpha)+2 A(\alpha)\right) .
\end{aligned}
$$

\footnotetext{
${ }^{9}$ Note that the existence of $K$ and $\gamma$ satisfying eq.(3.39) depends crucially on the asymptotic form (3.40) of $f(\alpha, n)$. This, in turn, is a non-trivial consequence of the specific form of the $n$-tachyon vertex given in eq.(3.7). For example, if $g^{(n)}$ had been momentum independent then we would get $b_{s}=\alpha / 2$ and $f(\alpha, n)=(n-1) \ln (2 \pi / \alpha)-\ln n$. This does not have the form (3.40).
} 
For a given value of $\alpha$, the above, together with $\beta=C(\alpha)$ (eqn. (3.38)) determine the functions $\psi(p)$ and $\chi(p)$ through eq. (3.24). These functions, in turn, generate a solution of the equations of motion of the form $\phi_{0} \chi(p)+$ $h(p)$ (eqn (3.20)).

As already pointed out, the natural interpretation of this solution is that of a $\left(N_{\|}-1\right)$-brane; we shall refer to this as a solitonic $\left(N_{\|}-1\right)$-brane. We would like to identify this with D- $\left(N_{\|}-1\right)$-brane. However, since there seems to be a one parameter family of solutions labelled by the parameter $\alpha$, we have the embarassment of riches. We shall now argue that the full solution $\phi_{0} \chi(p)+h(p)$ is independent of the choice of $\alpha$. To this end, note that using different values of $\alpha$ corresponds to moving along different paths in the complex $J(p)$ plane and returning to the origin. Indeed, we are considering the subspace of the full $J(p)$ space characterized by the parameters $\alpha$ and $u$ as follows:

$$
J(p)=u e^{-N_{\perp} A(\alpha) / 2} e^{-\alpha p_{\perp}^{2} / 2} \delta\left(p_{\|}\right) .
$$

In the complex $(u, \alpha)$ plane we go around $u=u_{b}$ and return to $u=0$ for a fixed $\alpha$. For $u=0$ which is the initial and final value of $u$, different $\alpha$ correspond to same $J(p)$, so the different paths characterized by different values of $\alpha$ have the same initial and final points. As we change $\alpha$ we change the path in the complex $(u, \alpha)$ space keeping fixed the initial and the final points. Since we do not cross any branch point during such deformation of the path, we should expect to have the same change in $\phi_{c}[p, J]$ for different $\alpha$. As a result the final solution $\phi_{0} \chi(p)+h(p)$ should be independent of $\alpha$, even though individually $\chi(p)$ and $h(p)$ depend on $\alpha$. In the absence of knowledge of the $h(p)$ term, however, we cannot determine the explicit form of the solution.

\subsection{Computation of ratios of lump tensions}

We shall now compute the tension associated with this solution and compare with the known tension of the $\mathrm{D}-\left(N_{\|}-1\right)$-brane. For this we need to evaluate the effective action $\Gamma[\phi]$ at $\phi=\phi_{0} \chi(p)+h(p)$. Since at the solution of the classical equations of motion $\Gamma[\phi]=-W[J]$, the value of the action for this solution is given by:

$$
\Gamma\left[\phi_{0} \chi(p)+h(p)\right]=-\lim _{u \rightarrow 0^{\prime}} W[u \psi(p)] .
$$

In taking the $u \rightarrow 0^{\prime}$ limit, we need to choose the branch in the complex $u$ plane along which $F(u)$ approaches $\phi_{0}$. Now from eq.(3.1) we see that on 
the trivial branch,

$$
W[u \psi(p)]=\sum_{n=2}^{\infty} \frac{u^{n}}{n !} \int\left(\prod_{k=1}^{n} d^{N} p_{k}\right) g^{(n)}\left(p_{1}, \ldots p_{n}\right) \psi\left(p_{1}\right) \ldots \psi\left(p_{n}\right) \delta\left(\sum_{i=1}^{n} p_{i}\right) .
$$

Using the form (3.7) for $g^{(n)}$ and (3.24) for $\psi(p)$, and explicitly performing the integration over the $p_{i \|}$, we get

$$
\begin{aligned}
W[u \psi(p)]=\delta_{\|}(0) \sum_{n=2}^{\infty} & \frac{1}{n !} C_{n} u^{n} K^{n} \int \prod_{k=1}^{n} d^{N_{\perp}} p_{k \perp} \\
& \cdot \delta\left(\sum_{i=1}^{n} p_{i \perp}\right) \exp \left(\sum_{k, l=1}^{n} a_{k-l} p_{k \perp} \cdot p_{l \perp}\right) .
\end{aligned}
$$

$a_{k}$ 's have been defined in eq.(3.27), and $\delta_{\|}(0)$ stands for $\delta\left(p_{\|}=0\right)$. Using the standard finite volume regularization we can identify $\delta_{\|}(0)$ with $V_{\|} /(2 \pi)^{N_{\|}}$, where $V_{\|}$is the total volume spanned by the coordinates $x_{\|}$. The integral appearing in eq.(3.48) is the same integral that appears in eq.(3.28) with $x_{\perp}$ set to zero. Performing the integral as before, we get

$$
W[u \psi(p)]=\frac{V_{\|}}{(2 \pi)^{N_{\|}}} \sum_{n=2}^{\infty} \frac{1}{n !} C_{n} u^{n} K^{n}\left\{\frac{1}{n}\left(\prod_{s=1}^{n-1} \frac{\pi}{b_{s}}\right)\right\}^{N_{\perp} / 2} .
$$

We now use eqs.(3.37), (3.40), (3.44) to write this as

$$
W[u \psi(p)]=\frac{V_{\|}}{(2 \pi)^{N_{\|}}} \sum_{n=2}^{\infty} \frac{1}{n !} C_{n} u^{n} \exp \left[\frac{N_{\perp}}{2}\left(B(\alpha)+s_{n}(\alpha)\right)\right],
$$

where the coefficients $s_{n}$, introduced in (3.40) approach zero as $n \rightarrow \infty$. If we define

$$
G(u)=\sum_{n=2}^{\infty} \frac{1}{n !} C_{n} u^{n}
$$

and

$$
P(u)=\exp \left(\frac{N_{\perp}}{2} B(\alpha)\right) \sum_{n=2}^{\infty} \frac{1}{n !} C_{n} u^{n}\left(e^{N_{\perp} s_{n}(\alpha) / 2}-1\right),
$$

then we can rewrite (3.50) as:

$$
W[u \psi(p)]=\frac{V_{\|}}{(2 \pi)^{N_{\|}}}\left[\exp \left(\frac{N_{\perp}}{2} B(\alpha)\right) G(u)+P(u)\right] .
$$


Since $\lim _{n \rightarrow \infty} s_{n}=0$, the coefficients of the Taylor series expansion in $u$ of $P(u)$ grows at a rate slower than that of $G(u)$.

Comparison with eq.(3.14) shows that $F(u)=d G(u) / d u$. Thus $G(u)$ must also have a branch point at $u=u_{b}$. On the other hand, since the Taylor series expansion of $P(u)$ converges faster than that of $G(u), P(u)$ has no branch point for $|u|<\left|u_{b}\right|$, and may or may not have a branch point at $u=u_{b}$. We now analytically continue $u$ around $u_{b}$ and return to the origin. Suppose during this process $G(u)$ returns to some constant $G_{0}$. It is important to note that $G_{0}$ does not depend on $N_{\perp}$, as is clear from eqn. (3.51). If $P(u)$ does not have a branch point at $u_{b}$ then it returns to zero, otherwise it returns to some value $P_{0}$. Thus using (3.46) and (3.53) we get

$$
\Gamma\left[\phi_{0} \chi(p)+h(p)\right]=-\frac{V_{\|}}{(2 \pi)^{N_{\|}}}\left(e^{N_{\perp} B(\alpha) / 2} G_{0}+P_{0}\right) .
$$

We shall refer to $P_{0}$ as the sub-leading contribution since it comes from the function $P(u)$ with a milder singularity at $u_{b}$ than $G(u)$. Note that $P(u)$ and hence $P_{0}$ vanishes for $N_{\perp}=0$. Thus if $P_{0}$ does not vanish it is necessarily $N_{\perp}$ dependent. We shall now make the final assumption in our analysis, namely that the sub-leading contribution $P_{0}$ vanishes. ${ }^{10}$ Using this critical assumption in eq.(3.54), we can identify the tension of the solitonic $\left(N_{\|}-1\right)$ brane to be:

$$
\mathcal{T}_{N_{\|}-1}=\frac{1}{(2 \pi)^{N_{\|}}} G_{0} e^{\left(N-N_{\|}\right) B(\alpha) / 2},
$$

where we have used $N=N_{\|}+N_{\perp}$. We now note that the function $G(u)$ and hence the constant $G_{0}$ does not depend on $N_{\perp}$. Thus eq.(3.55) gives the prediction:

$$
\frac{1}{2 \pi} \frac{\mathcal{T}_{N_{\|}-2}}{\mathcal{T}_{N_{\|}-1}}=e^{B(\alpha) / 2}
$$

Using the numerical result that $B(\alpha)$ vanishes identically, we see that the right hand side of eq.(3.56) becomes 1 . This is in perfect agreement with the exact answer.

With our present knowledge, however, we cannot prove that $P_{0}$ vanishes. Nevertheless, the remarkable agreement described above can be taken as

\footnotetext{
${ }^{10}$ This is not in contradiction with the earlier claim (previous subsection) that the subleading contribution $h(q)$ to the classical solution must be non-vanishing so that $\phi_{0} \chi(q)+$ $h(q)$ is independent of $\alpha$. Indeed, $P_{0}$ receives contribution only from the sub-leading terms $s_{n}$ in $f(\alpha, n)$, whereas $h(q)$ receives contributions also from the sub-leading terms in $k(\alpha, n)$.
} 
an evidence for the underlying assumptions in our analysis, namely that the quadratic term in the string field theory action expanded around the tachyon vacuum is made solely of the ghost operators, and also that the non-leading contribution from the function $P(u)$ vanishes at $u=0$. Further analysis of the non-leading terms $s_{n}$ is required to establish if $P_{0}$ vanishes. In fact, even the knowledge of $C_{n}$ may be important for this analysis, in which case a specific choice of the kinetic operator would be required.

\section{Further studies and open questions}

In this section we discuss important issues for which our understanding is fairly incomplete. We begin by exploring how much freedom there is in choosing a kinetic operator built exclusively from the ghost sector. Then we discuss some attempts to use level expansion to understand the proposed actions around the tachyon vacuum. We discuss some difficulties with the use of the Siegel gauge, and some alternative gauges. Finally we give some remarks on extensions to superstring theory, and on implications for the search of closed strings states.

\subsection{On the uniqueness of $\mathcal{Q}$}

Our discussion of kinetic operators $\mathcal{Q}$ that are constructed purely of ghost operators identified a family of them, and thus the general form in (2.19) $\mathcal{Q}=\sum_{n \geq 0} a_{n} \mathcal{C}_{n}$. More general $\mathcal{Q}$ 's made of purely ghost operators might exist, but we will focus here on this family.

The key question is whether all these operators are actually equivalent. If they are, anyone of them could be chosen, making $\mathcal{Q}=c_{0}$ the most obvious choice. If they are not equivalent, one must search for the correct one.

As discussed in section 2.2 and in particular in equations (2.12), field redefinitions preserving the cubic structure of the theory arise from BPZ odd ghost number zero operators that are derivations of the star algebra. There is a well-known family of such operators, they are conformal transformations that leave the string midpoint fixed [75]

$$
K_{n} \equiv L_{n}-(-1)^{n} L_{-n}, \quad n \geq 1 .
$$

With $\mathcal{C}_{n}=c_{n}+(-)^{n} c_{-n}$ we have the algebra

$$
\left[K_{n}, \mathcal{C}_{m}\right]=-\left[(2 n+m) \mathcal{C}_{m+n}+(-)^{n}(2 n-m) \mathcal{C}_{m-n}\right] .
$$


For example $\left[K_{n}, \mathcal{C}_{0}\right]=-4 n \mathcal{C}_{n}$, and this indicates that the operator $\mathcal{Q}=\mathcal{C}_{0}$ can be deformed infinitesimally in every direction except along itself by a field redefinition of the form $\Psi \rightarrow \exp \left(\epsilon_{n} K_{n}\right) \Psi$. A change of $\mathcal{Q}$ along itself corresponds to a change in coupling, and we do not expect such changes to be possible by field redefinitions.

For more general $\mathcal{Q}$, even the infinitesimal deformation problem is somewhat nontrivial. Consider an arbitrary $\mathcal{Q}=\sum_{n \geq 0} a_{n} \mathcal{C}_{n}$ and an arbitrary deformation into $\mathcal{Q}^{\prime}=\mathcal{Q}+\epsilon \Delta \mathcal{Q}$ with $\Delta \mathcal{Q}=\sum_{n>0} e_{n} \mathcal{C}_{n}$, where only a finite number of $e_{n}$ 's are nonzero. We ask if $\mathcal{Q}$ and $\overline{\mathcal{Q}}^{\prime}$ are equivalent up to an infinitesimal scaling. With $K=\sum_{n} d_{n} K_{n}$ such equivalence will hold if there exist constants $d_{n}$ 's and a constant $r$ such that

$$
e^{\epsilon K} \mathcal{Q} e^{-\epsilon K}=(1+\epsilon r) \mathcal{Q}^{\prime} \quad \rightarrow \quad[K, \mathcal{Q}]=r \mathcal{Q}+\Delta \mathcal{Q} .
$$

If the solution involves an infinite number of non-vanishing $d_{n}$ 's, one must study their large $n$ behaviour to decide if the infinitesimal conformal transformation associated to $K$ exists. While it follows from the earlier remarks that for $\mathcal{Q}=c_{0}$ all infinitesimal deformations with $e_{0}=0$ yield equivalent kinetic operators, we suspect that this will not be the case for general $\mathcal{Q}$. One way to see this is to recall that the identity string field $\langle\mathcal{I}|$ is not annihilated by $c_{0}[15]$ :

$$
\langle\mathcal{I}| c_{0} \neq 0 .
$$

This is some sort of anomaly, since any derivation of the star product, such as $c_{0}$, would be expected to annihilate the identity of the product. On the other hand ([15], eqn. (6.16))

$$
\langle\mathcal{I}|\left(c_{0}+\frac{1}{2}\left(c_{2}+c_{-2}\right)\right)=0 .
$$

Since $\langle\mathcal{I}| K_{n}=0$ for all $n$, we cannot expect that

$$
\left(\mathcal{C}_{0}+\mathcal{C}_{2}+\epsilon \mathcal{C}_{0}\right) \sim \exp \left(\sum d_{n} K_{n}\right)\left(\mathcal{C}_{0}+\mathcal{C}_{2}\right) \exp \left(-\sum d_{n} K_{n}\right)
$$

with the exponentials defining regular transformations. This is clear since the right hand side would annihilate the identity while the left hand side would not.

It would therefore appear that up to rescaling there are at least two classes of inequivalent kinetic terms, those which annihilate the identity and those which do not. In fact, ref. [49] restricts its attention to those operators that annihilate the identity, as this string field plays an important role in the conjectured purely cubic form of SFT. 


\subsection{On the use of level truncation}

For the standard cubic open string field theory with the usual BRST operator $Q_{B}$, there is overwhelming numerical evidence (but yet no proof) that the level truncation scheme [9] provides results that converge to the correct nonperturbative answer. It is therefore of interest to ask whether level truncation can be used to test possible choices for the BRST operator $\mathcal{Q}$ around the tachyon vacuum. So far we have only obtained some partial and somewhat problematic results for the simplest choice $\mathcal{Q}=\tilde{A} c_{0}$. We allow for the presence of an unknown normalization constant $\tilde{A}$. By a rescaling of the string field, we choose to write $\mathcal{Q}=c_{0}$ and put an overall costant $A=\widetilde{A}^{3}$ in front of the string field theory action.

We first look for a translationally invariant solution of the truncated string field theory which corresponds to the space filling D-25 brane - the original vacuum before condensation. The potential for a static configuration is

$$
-S(\Psi)=\frac{1}{g_{0}^{2} 2 \pi^{2}} \mathcal{V}(\Psi)
$$

with

$$
\mathcal{V}(\Psi)=A \cdot 2 \pi^{2}\left[\frac{1}{2}\left\langle\Psi, c_{0} \Psi\right\rangle+\frac{1}{3}\langle\Psi, \Psi * \Psi\rangle\right] .
$$

By construction, the zero of the energy corresponds to the tachyon vacuum $\Psi=0, \mathcal{V}(\Psi=0)=0$ and all physical excitations are expected to have positive energy above this ground state. We adopt the normalization conventions of [76], in which the space filling solution $\Psi_{D_{25}}$ obeys $\mathcal{V}\left(\Psi_{D_{25}}\right)=1$. This in turn should allow to fix the constant $A$.

We work in the Siegel gauge $b_{0} \Psi=0$. In the level $(0,0)$ approximation, the effect of replacing $Q_{B}$ by $c_{0}$ is simply to change the sign of the kinetic term. From the results in [10] we readily find the translationally invariant solution $\Psi^{(0,0)}=t_{c} c_{1}|0\rangle$ with $t_{c}=-(4 \sqrt{3} / 9)^{3}$. This gives $\mathcal{V}^{(0,0)} \approx 0.684 A$. We simply quote the results that we find at higher levels: $\mathcal{V}^{(2,6)} \approx 0.1849 \mathrm{~A}$, $\mathcal{V}^{(4,8)} \approx 0.09656 A$. Unlike the case of the action with the standard BRST operator $Q_{B}$, here we do not find any evidence of convergence to a finite limit in the level truncation. Rather, the energy of the space filling brane seems to be pushed towards zero as the level is increased.

We have also looked for solitonic solutions $\Psi_{D_{24}}$ corresponding to D-24 branes, in which the string field has the shape of a lump along a direction $X$ and approaches asymptotically the tachyon vacuum. As in [14], in order 
to implement systematically the level truncation scheme we compactify the direction $X$ on a circle of radius $R$. For each zero momentum state $\left|\Psi_{i}\right\rangle$ that we had before, we obtain a tower of Fourier modes $\left|\Psi_{i, n}\right\rangle, n=0,1,2 \ldots$, of momentum $p=n / R$ along the $X$ direction. Level is now defined as $n^{2} / R^{2}+N_{i}-N_{0}$, where $N_{i}$ is the number operator and $N_{0}=-1$ is the number eigenvalue of the zero momentum tachyon.

We have considered the case $R=\sqrt{3}$ and performed the computation up to level $(3,6)$. As for finite $A$ the energy of the D25-brane appears to go to zero in our calculations, we considered an energy ratio where this fact would not affect the result. Indeed, the ratio

$$
\mathcal{R}^{(N, M)}=\frac{R \mathcal{V}\left(\Psi_{D_{24}}^{(N, M)}\right)}{\mathcal{V}\left(\Psi_{D_{25}}^{(N, M)}\right)},
$$

does not depend on the unknown constant $A$ and is expected to converge to 1 . We find: $\mathcal{R}^{(1 / 3,2 / 3)} \approx 1.11056, \mathcal{R}^{(4 / 3,8 / 3)} \approx 0.958355, \mathcal{R}^{(7 / 3,14 / 3)} \approx 1.07661$, $\mathcal{R}^{(3,6)} \approx 1.2141$. Although not far from the expected answer, it is not clear we are finding convergence to the correct value.

The conclusion is that level truncation with $\mathcal{Q}=c_{0}$ and in the Siegel gauge is somewhat problematic. This could be (i) a general problem with level expansion around the tachyon vacuum whenever the kinetic term has been brought to a purely ghost form, (ii) a problem with the specific choice $\mathcal{Q}=c_{0}$ as opposed to other choices in the general family $\mathcal{Q}=\sum a_{n} \mathcal{C}_{n}$, or, (iii) a problem with the Siegel gauge. Indeed, there are reasons to believe that this may not be a legal choice, as we shall discuss in the next subsection.

\subsection{On gauge fixing}

Our analysis of the tachyon effective action in section 3 assumes that given a string field theory with a purely ghost BRST operator $\mathcal{Q}$, one can find a suitable gauge fixing which does not involve matter operators. We believe on general grounds that this should always be possible. However we would like to point out some complications which arise in the application of the standard Siegel gauge to string field theories with non-standard BRST operators, like the family $\mathcal{Q}=\sum_{n \geq 0} a_{n} \mathcal{C}_{n}$.

We focus again on the simplest case of the string field theory with $\mathcal{Q}=c_{0}$. Let us consider imposing the Siegel gauge condition $b_{0} \Psi=0$. This is always possible at the linearized level, since if $b_{0} \Psi \neq 0$, then $\Psi_{S}=\Psi-\mathcal{Q}\left(b_{0} \Psi\right)=$ $\Psi-c_{0} b_{0} \Psi$ satisfies $b_{0} \Psi_{S}=0$. Moreover, the Siegel condition fixes the gauge 
completely. Indeed if $\delta \Psi$ is pure gauge, and in Siegel gauge, then $\mathcal{Q} \delta \Psi=0$ and $b_{0} \delta \Psi=0$, leading necessarily to $\delta \Psi=0 .{ }^{11}$

Consider now computing perturbative amplitudes in this string field theory. We shall find that tree level Green's functions diverge in the Siegel gauge. The only modification of the conventional Feynman rules [41] is that the propagator is $b_{0}$ instead of $b_{0} L_{0}^{-1}$. This is however a dramatic difference. The factor $L_{0}^{-1}=\int_{0}^{\infty} e^{-t L_{0}}$ had the interpretation of an integral over strips of any length. In the present case, the strip has collapsed to zero length, and there is no integration over moduli space, but simply a ghost insertion $b_{0}=\int d \sigma\left(b_{z z}+b_{\bar{z} \bar{z}}\right)$ across the collapsed strip. Any term in the $n$-point amplitude requires $(n-3)$ ghost insertions of the form

$$
\int_{\gamma / 2}\left(d z b\left(f_{k}^{(n)}(z)\right) z\left(\frac{d f_{k}^{(n)}}{d z}\right)^{2}+a . h .\right)
$$

where $f_{k}^{(n)}(z)$ were defined in (3.4). Each integral across a collapsed strip has been referred to the local coordinates $z$ of the relevant half-strings. One of these contributions is what is shown in the above formula. Thus $\gamma / 2$ means either the right or the left half-boundary of a canonical upper-half unit disk. In addition, the $b(z)$ appearing in this half-string integral has been mapped into the global uniformizing disk where all the strings are glued together. This is necessary because all correlators are computed on the uniformizing disk. Near $z=i$ the integrand behaves as $\sim(1+i z)^{-2+4 / n}$, which is a non-integrable singularity for $n \geq 4$. Thus in Siegel gauge, the coefficients $C_{n}$ in (3.2) appear to diverge.

There is a simple cure for this problem, which consists of considering a gauge-fixing condition $B \Psi=0$ with $B=\oint z g(z) b(z)$, where $g(z) \sim(1+i z)^{m}$ near $z \sim i$, and $m>1$. We also demand that $g(z)$ contains a constant in its Laurent expansion, so that $B$ has some amount of $b_{0}$ : this is required to have $\left\{B, c_{0}\right\} \neq 0$ so that the gauge condition can be imposed (at least at the linearized level). For example, with $g(z)=1+\left(z^{2}+1 / z^{2}\right) / 2$ we have $B=b_{0}+\left(b_{2}+b_{-2}\right) / 2$. The extra double zero $\sim(1+i z)^{2}$ of the propagator near $z=i$ ensures now the convergence of string amplitudes. It would be interesting to explore these 'finite' gauge choices in the level truncation scheme.

\footnotetext{
${ }^{11}$ As long as $L_{0} \Psi \neq 0$, this analysis is entirely parallel to the one with $Q_{B}$, for which $\left\{b_{0}, Q_{B}\right\}=L_{0}$.
} 


\subsection{On superstring field theory and closed strings}

As was the case for the bosonic theory we may conjecture that at the tachyon vacuum the kinetic term of superstring field theory does not involve a matter sector operator. We will offer some remarks that may help in constructing an appropriate action.

The algebraic structure of the open Neveu-Schwarz (NS) superstring field theory of refs. $[55,56,57]$ demands the existence of two Grassmann odd operators $Q_{B}$ and $\eta_{0}$ anticommuting with each other, both of which square to zero, satisfy the same BPZ conjugation property, and are derivations of the star algebra. The operator $\eta_{0}$, having picture number minus one, is the zero mode of the Grassmann odd field $\eta(z)$ arising from fermionization of the superghosts. If the BRST operator $Q_{B}$ is replaced by $c_{0}$ or any of the $\mathcal{C}_{n}$ operators, the algebraic structure will remain in place, for this operator would still commute with $\eta_{0}$ and, as we have discussed before, satisfies all the other requisite properties. Thus an action with $\mathcal{Q}=\sum_{n} a_{n} \mathcal{C}_{n}$ would satisfy the conditions of gauge invariance. Moreover, there would be no physical states around this vacuum. Indeed, in this string field theory the linearized equations of motion are $\mathcal{Q} \eta_{0} \Phi=0$ where $\Phi$ is a string field of ghost number zero and picture number zero in the so-called large Hilbert space. The gauge invariance $\delta \Phi=\eta_{0} \Omega$ allows one to write $\Phi=\xi_{0} V$ where $V$ is a conventional picture number minus one field of the NS sector. In this partial gauge the equation of motion reduces to $\mathcal{Q} V=0$ which, for $\mathcal{Q}=Q_{B}$ is the standard BRST cohomology problem in the conventional small Hilbert space. If $\mathcal{Q}=\sum a_{n} \mathcal{C}_{n}$, we will have no physical states.

While it seems tempting to simply replace $Q_{B}$ by the appropriate ghost operator to obtain an action around the tachyon vacuum, there are some questions that suggest that this change may not suffice to obtain the desired action. In particular, the Chan-Paton like factors that implement the $Z_{2}$ symmetry under which the GSO odd states change sign [64,65] must somehow change in the tachyon vacuum. Indeed, while the tachyon potential is even with respect to the field variable which vanishes at the unstable vacuum, it is not even with respect to the field variable which vanishes at any one of the tachyon vacua.

It is perhaps worthwhile to note that the replacement $Q_{B} \rightarrow \sum a_{n} \mathcal{C}_{n}$ does not preserve the algebraic structure of cubic superstring field theory [75]. The problem is that the picture changing operator $X(z)$, that must be inserted at the string midpoint in the definition of the star product, while BRST invariant, is not $\mathcal{C}_{n}$ invariant. This spoils the derivation property 
which is necessary for gauge invariance. This cubic string field theory, in any case, has long been known to be problematic [77], and does not appear to give a sensible tachyon potential either [78]. Interestingly, the double picture changing insertion used in the cubic superstring field theory action advocated in refs. $[69,70,72]$ does commute with the $\mathcal{C}_{n}$ operators, and it therefore appears that gauge invariant actions without cohomology could be constructed.

We can now ask if a similar replacement of the BRST operator with another having trivial cohomology can be done in closed string field theory [80]. The answer is no. The problem here is that the interactions beyond the cubic one involve the integration of correlators over finite pieces of the moduli spaces of Riemann surfaces. Key to the algebraic structure of the theory is the fact that the BRST operator acts as a total derivative on moduli space. This arises because forms on moduli space involve the antighost field $b(z)$ and $\left\{Q_{B}, b(z)\right\}=T(z)$, where the energy momentum tensor $T$ generates deformations of correlators. Such property will not hold upon replacing $Q_{B}$ with an object constructed purely from ghosts.

A final question is whether closed strings arise from the kind of open string field theory we have been considering here, namely one with a kinetic opertor having no cohomology. We have no definite answer. Recall that in cubic open string field theory closed string poles definitely arise in string loop amplitudes [81]. A subset of open string propagators in a string diagram, represented by a strip of total length $T \in[0, \infty]$, whose middle line defines a nontrivial closed curve, will give rise to closed string poles via integration over the neighborhood of $T=0$. In the kind of actions we have considered here, the propagator collapses to an overlap with some antighost insertion. It does not seem possible to obtain closed string poles from an amplitude that just includes the contribution from the point $T=0$. On the other hand, there are well known complications with the precise BatalinVilkovisky quantization of conventional cubic open string field theory, and they precisely arise from the $T \rightarrow 0$ limit of a one-point one-loop amplitude $[82,83]$. The whole issue surrounding closed strings in open string field theory after tachyon condensation is subtle enough $[84,85,86,87,88]$ that further work seems necessary to attain definite conclusions.

\subsection{Concluding remarks}

The open string field theories studied in this paper manifestly implement the absence of conventional open string dynamics on the vacuum of the tachyon. 
They naturally implement gauge invariance and are rather reminiscent of the $p$-adic string theory, where after a simple invertible field redefinition the kinetic operator is just a constant. In addition, they are in many ways strikingly simple, much more so than the conventional cubic open string field theory in the background of a D-brane. For example, while we were able here to compute the exact momentum dependence of the off-shell $n$ tachyon amplitudes, such computation appears to be prohibitively complicated in conventional cubic string field theory. If the normalization of the $n$-tachyon amplitudes can be computed, perhaps along the lines of comments in sect. 4.3 , we may then be able to find the explicit solutions representing D-branes, to obtain the precise form of the kinetic term $\mathcal{Q}$, and to settle the outstanding assumptions of our analysis.

Acknowledgements: We would like to thank D. Ghoshal, D. Jatkar, N. Moeller, P. Mukhopadhyay, S. Panda, and W. Taylor for useful discussions. The work of L.R. was supported in part by Princeton University "Dicke Fellowship" and by NSF grant 9802484 . The work of B.Z. was supported in part by DOE contract \#DE-FC02-94ER40818.

\section{References}

[1] A. Sen, Descent relations among bosonic D-branes, Int. J. Mod. Phys. A14, 4061 (1999) [hep-th/9902105].

[2] A. Sen, Non-BPS states and branes in string theory, hep-th/9904207.

[3] A. Recknagel and V. Schomerus, Boundary deformation theory and moduli spaces of D-branes, Nucl. Phys. B545, 233 (1999) [hepth/9811237].

[4] C.G. Callan, I.R. Klebanov, A.W. Ludwig and J.M. Maldacena, Exact solution of a boundary conformal field theory, Nucl. Phys. B422, 417 (1994) [hep-th/9402113].

[5] J. Polchinski and L. Thorlacius, Free fermion representation of a boundary conformal field theory, Phys. Rev. D50, 622 (1994) [hepth/9404008].

[6] P. Fendley, H. Saleur and N.P. Warner, Exact solution of a massless scalar field with a relevant boundary interaction, Nucl. Phys. B430, 577 (1994) [hep-th/9406125]. 
[7] J. Harvey, D. Kutasov and E.J. Martinec, On the relevance of tachyons, [hep-th/0003101].

[8] S. Dasgupta and T. Dasgupta, Renormalization group analysis of tachyon condensation, hep-th/0010247.

[9] V.A. Kostelecky and S. Samuel, The Static Tachyon Potential in the Open Bosonic String Theory, Phys. Lett. B207 (1988) 169.

[10] A. Sen and B. Zwiebach, Tachyon Condensation in String Field Theory, JHEP 0003, 002 (2000) [hep-th/9912249].

[11] N. Moeller and W. Taylor, Level truncation and the tachyon in open bosonic string field theory, Nucl. Phys. B583, 105 (2000) [hepth/0002237].

[12] J.A. Harvey and P. Kraus, D-Branes as unstable lumps in bosonic open string field theory, JHEP 0004, 012 (2000) [hep-th/0002117].

[13] R. de Mello Koch, A. Jevicki, M. Mihailescu and R. Tatar, Lumps and p-branes in open string field theory, Phys. Lett. B482, 249 (2000) [hepth/0003031].

[14] N. Moeller, A. Sen and B. Zwiebach, D-branes as tachyon lumps in string field theory, hep-th/0005036.

[15] L. Rastelli and B. Zwiebach, Tachyon potentials, star products and universality, hep-th/0006240.

[16] A. Sen and B. Zwiebach, Large marginal deformations in string field theory, JHEP 0010, 009 (2000) [hep-th/0007153].

[17] W. Taylor, Mass generation from tachyon condensation for vector fields on D-branes, JHEP 0008, 038 (2000) [hep-th/0008033].

[18] I. Ellwood and W. Taylor, private communication and work in progress.

[19] R. de Mello Koch and J.P. Rodrigues, Lumps in level truncated open string field theory, hep-th/0008053.

[20] N. Moeller, Codimension two lump solutions in string field theory and tachyonic theories, hep-th/0008101.

[21] A. Kostelecky and R. Potting, Analytical construction of a nonperturbative vacuum for the open bosonic string, hep-th/0008252.

[22] H. Hata and S. Shinohara, BRST invariance of the non-perturbative vacuum in bosonic open string field theory, JHEP 0009, 035 (2000) [hep-th/0009105]. 
[23] B. Zwiebach, Trimming the Tachyon String Field with $S U(1,1)$, hepth/0010190.

[24] M. Schnabl, Constraints on the tachyon condensate from anomalous symmetries, hep-th/0011238.

[25] R. Gopakumar, S. Minwalla and A. Strominger, Noncommutative solitons, JHEP 0005, 020 (2000) [hep-th/0003160].

[26] K. Dasgupta, S. Mukhi and G. Rajesh, Noncommutative tachyons, JHEP 0006, 022 (2000) [hep-th/0005006].

[27] J. A. Harvey, P. Kraus, F. Larsen and E. J. Martinec, D-branes and strings as non-commutative solitons, hep-th/0005031.

[28] E. Witten, Noncommutative tachyons and string field theory, hepth/0006071.

[29] J. A. Harvey, P. Kraus and F. Larsen, Exact noncommutative solitons, hep-th/0010060.

[30] G. Mandal and S. Rey, A note on D-branes of odd codimensions from noncommutative tachyons, Phys. Lett. B495, 193 (2000) [hepth/0008214].

[31] A.A. Gerasimov and S.L. Shatashvili, On exact tachyon potential in open string field theory, hep-th/0009103.

[32] D. Kutasov, M. Marino and G. Moore, Some exact results on tachyon condensation in string field theory, hep-th/0009148.

[33] D. Ghoshal and A. Sen, Normalisation of the background independent open string field theory action, hep-th/0009191.

[34] J. R. David, Tachyon condensation using the disc partition function, hep-th/0012089.

[35] D. Ghoshal and A. Sen, Tachyon condensation and brane descent relations in p-adic string theory, Nucl. Phys. B584, 300 (2000) [hepth/0003278].

[36] B. Zwiebach, A solvable toy model for tachyon condensation in string field theory, JHEP 0009, 028 (2000) [hep-th/0008227].

[37] J.A. Minahan and B. Zwiebach, Field theory models for tachyon and gauge field string dynamics, JHEP 0009, 029 (2000) [hep-th/0008231]. 
[38] J. A. Minahan and B. Zwiebach, Effective tachyon dynamics in superstring theory, hep-th/0009246.

[39] J. A. Minahan and B. Zwiebach, Gauge fields and fermions in tachyon effective field theories, hep-th/0011226.

[40] G. Arutyunov, S. Frolov, S. Theisen and A. A. Tseytlin, Tachyon condensation and universality of DBI action, hep-th/0012080.

[41] E. Witten, Noncommutative Geometry And String Field Theory, Nucl. Phys. B268, 253 (1986).

[42] E. Witten, On background independent open string field theory, Phys. Rev. D46, 5467 (1992) [hep-th/9208027].

[43] E. Witten, Some computations in background independent off-shell string theory, Phys. Rev. D47, 3405 (1993) [hep-th/9210065].

[44] K. Li and E. Witten, Role of short distance behavior in off-shell open string field theory, Phys. Rev. D48, 853 (1993) [hep-th/9303067].

[45] S.L. Shatashvili, Comment on the background independent open string theory, Phys. Lett. B311, 83 (1993) [hep-th/9303143].

[46] S.L. Shatashvili, On the problems with background independence in string theory, hep-th/9311177.

[47] A. A. Tseytlin, Sigma model approach to string theory effective actions with tachyons, hep-th/0011033.

[48] E. Witten, unpublished.

[49] G. T. Horowitz, J. Morrow-Jones, S. P. Martin and R. P. Woodard, New Exact Solutions For The Purely Cubic Bosonic String Field Theory, Phys. Rev. Lett. 60, 261 (1988).

[50] G. T. Horowitz, J. Lykken, R. Rohm and A. Strominger, A Purely Cubic Action For String Field Theory, Phys. Rev. Lett. 57, 283 (1986).

[51] H. Hata, K. Itoh, T. Kugo, H. Kunitomo and K. Ogawa, "Pregeometrical String Field Theory: Creation Of Space-Time And Motion," Phys. Lett. B175, 138 (1986).

[52] R. Gopakumar, S. Minwalla and A. Strominger, Symmetry restoration and tachyon condensation in open string theory, hep-th/0007226.

[53] N. Seiberg, A note on background independence in noncommutative gauge theories, matrix model and tachyon condensation, hepth/0008013. 
[54] A. Sen, Some issues in non-commutative tachyon condensation, hepth/0009038.

[55] N. Berkovits, Super-Poincare Invariant Superstring Field Theory, Nucl. Phys. B450 (1995) 90, [hep-th/9503099].

[56] N. Berkovits and C.T. Echevarria, Four-Point Amplitude from Open Superstring Field Theory, [hep-th/9912120].

[57] N. Berkovits, A New Approach to Superstring Field Theory, proceedings to the $32^{n d}$ International Symposium Ahrenshoop on the Theory of Elementary Particles, Fortschritte der Physik (Progress of Physics) 48 (2000) 31, [hep-th/9912121].

[58] A. Sen, Stable nonBPS bound states of BPS D-branes, JHEP 08, 010 (1998) hep-th/9805019.

[59] A. Sen, Tachyon condensation on the brane anti-brane system, JHEP 08, 012 (1998) hep-th/9805170.

[60] A. Sen, SO(32) spinors of type I and other solitons on brane - anti-brane pair, JHEP 09, 023 (1998) hep-th/9808141.

[61] E. Witten, D-branes and $K$ theory, JHEP 12, 019 (1998) hepth/9810188.

[62] A. Sen, BPS D branes on nonsupersymmetric cycles, JHEP 12, 021 (1998) hep-th/9812031.

[63] P. Horava, Type IIA D-branes, $K$ theory, and matrix theory, hepth/9812135.

[64] N. Berkovits, The Tachyon Potential in Open Neveu-Schwarz String Field Theory, [hep-th/0001084].

[65] N. Berkovits, A. Sen and B. Zwiebach, Tachyon condensation in superstring field theory, hep-th/0002211.

[66] P. De Smet and J. Raeymaekers, Level four approximation to the tachyon potential in superstring field theory, hep-th/0003220.

[67] A. Iqbal and A. Naqvi, Tachyon condensation on a non-BPS D-brane, hep-th/0004015.

[68] L. Brekke, P. G. Freund, M. Olson and E. Witten, Nonarchimedean String Dynamics, Nucl. Phys. B302, 365 (1988). 
[69] I. Y. Arefeva, P. B. Medvedev and A. P. Zubarev, Background Formalism For Superstring Field Theory, Phys. Lett. B240, 356 (1990).

[70] I. Y. Arefeva, P. B. Medvedev and A. P. Zubarev, New Representation For String Field Solves The Consistence Problem For Open Superstring Field, Nucl. Phys. B341, 464 (1990).

[71] C. R. Preitschopf, C. B. Thorn and S. A. Yost, Superstring Field Theory, Nucl. Phys. B337, 363 (1990).

[72] I. Y. Aref'eva, D. M. Belov, A. S. Koshelev and P. B. Medvedev, Tachyon condensation in cubic superstring field theory, hep-th/0011117.

[73] A. Sen and B. Zwiebach, A proof of local background independence of classical closed string field theory, Nucl. Phys. B414, 649 (1994) [hepth/9307088].

[74] T. Kawano and T. Takahashi, Open string field theory on noncommutative space, hep-th/9912274.

[75] E. Witten, Interacting Field Theory Of Open Superstrings, Nucl. Phys. B276, 291 (1986).

[76] A. Sen, Universality of the tachyon potential, JHEP 9912, 027 (1999) [hep-th/9911116].

[77] C. Wendt, Scattering amplitudes and contact interactions in Witten's superstring field theory, Nucl. Phys. B 314209 (1989).

J. Greensite and F.R. Klinkhamer, Superstring Amplitudes and Contact Interactions, Nucl. Phys. B304 (1988) 108.

[78] P. De Smet and J. Raeymaekers, The tachyon potential in Witten's superstring field theory, JHEP 0008, 020 (2000) [hep-th/0004112].

[79] S. Giddings, E. Martinec and E. Witten, Modular invariance in string field theory, Phys. Lett. B226 362 (1986).

B. Zwiebach, A proof that Witten's open string theory gives a single cover of moduli space, Commun. Math. Phys. 142193 (1991).

[80] B. Zwiebach, Closed string field theory: Quantum action and the BV master equation. Nucl.Phys. B390(1993) 33-152, hep-th/9206084.

[81] D. Z. Freedman, S. B. Giddings, J. A. Shapiro and C. B. Thorn, "The Nonplanar One Loop Amplitude In Witten's String Field Theory," Nucl. Phys. B298, 253 (1988). 
[82] C. B. Thorn, String field theory, Phys. Rep. 174 (1989) 1;

A. R. Bogojevic, BRST Invariance of the measure in string field theory, Phys. Lett. B198 (1987) 479;

M. Maeno, Canonical quantization of Witten's string field theory using midpoint light cone time, Phys. Rev. D43 (1991) 4006.

[83] B. Zwiebach, Oriented open-closed string theory revisited, Annals Phys. 267, 193 (1998) [hep-th/9705241].

[84] M. Kleban, A. Lawrence and S. Shenker, Closed strings from nothing, hep-th/0012081.

[85] P. Yi, Membranes from five-branes and fundamental strings from $D p$ branes, Nucl. Phys. B550, 214 (1999) [hep-th/9901159].

[86] O. Bergman, K. Hori and P. Yi, Confinement on the brane, Nucl. Phys. B580, 289 (2000) [hep-th/0002223].

[87] G. Gibbons, K. Hori and P. Yi, String fluid from unstable D-branes, hep-th/0009061.

[88] A. Sen, Fundamental strings in open string theory at the tachyonic vacuum, hep-th/0010240. 